\title{
Corticosterone Signaling and a Lateral Habenula-Ventral Tegmental Area Circuit Modulate Compulsive Self-Injurious Behavior in a Rat Model
}

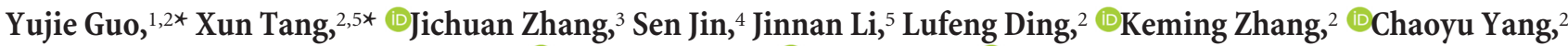 \\ Hua Zhou, ${ }^{3}$ Xiaobin He, ${ }^{4}$ Fuqiang Xu, ${ }^{4}$ Guo-Qiang Bi, ${ }^{1,2} \oplus^{-}$Lin $\mathrm{Xu},{ }^{5}$ and $\odot$ Pak-Ming Lau ${ }^{2}$ \\ ${ }^{1}$ Hefei National Laboratory for Physical Sciences at the Microscale, ${ }^{2}$ Chinese Academy of Sciences Key Laboratory of Brain Function and Disease, School of \\ Life Sciences, University of Science and Technology of China, Hefei, Anhui 230027, China, ${ }^{3}$ Faculty of Medicine, Kunming University of Science and \\ Technology, Kunming, Yunnan 650500, China, ${ }^{4}$ State Key Laboratory of Magnetic Resonance and Atomic and Molecular Physics and Center for Excellence \\ in Brain Science and Intelligence Technology, Wuhan Institute of Physics and Mathematics, Chinese Academy of Sciences, Wuhan, Hubei 430071, China, \\ and ${ }^{5}$ Key Laboratory of Animal Models and Human Disease Mechanisms, Laboratory of Learning and Memory, Center for Excellence in Brain Science and \\ Intelligence Technology, Kunming Institute of Zoology, Chinese Academy of Sciences, Kunming, Yunnan 650223, China
}

Self-injurious behavior (SIB) is commonly observed in patients with neuropsychiatric disorders, as well as in nonclinical populations with stress-related mental-health problems. However, the exact circuitry mechanisms underlying SIB have remained poorly understood. Here, with bilateral injection of muscimol into the entopeduncular nucleus (EP), we established a rat model of SIB. Following the muscimol injection, the male rats exhibited in a dose-dependent manner stereotypic self-biting behavior that lasted for hours and often resulted in wounds of various severities. The SIB was associated with an elevated level of serum corticosterone and could be exacerbated by enhancing the corticosterone signaling and, conversely, alleviated by inhibiting the corticosterone signaling. Activity mapping using c-fos immunostaining, combined with connectivity mapping using herpes simplex virus-based anterograde tracing from the EP and pseudorabies virus-based retrograde tracing from the masseter muscle, revealed the potential involvement of many brain areas in SIB. In particular, the lateral habenula (LHb) and the ventral tegmental area (VTA), the two connected brain areas involved in stress response and reward processing, showed a significant increase in neuronal activation during SIB. Furthermore, suppressing the LHb activity or modulating the GABAergic transmission in the VTA could significantly reduce the occurrence of SIB. These results demonstrate the importance of stress hormone signaling and the LHb-VTA circuit in modulating SIB resulting from EP malfunction, and suggest potential targets for therapeutic intervention of SIB and related disorders.

Key words: entopeduncular nucleus; glucocorticoid; lateral habenula; self-injurious behavior; stress; ventral tegmental area

Significance Statement

Self-injurious behavior (SIB) occurs in $\sim 4 \%$ of the general population, with substantially higher occurrence among adolescents and patients of neuropsychiatric disorders. Stress has been linked to the occurrence of SIB, yet the underlying mechanisms have remained unclear. Using a rat model of SIB induced by disruption of activity in the entopeduncular nucleus (EP), we found that the behavior is regulated by stress and linked to corticosterone signaling. Viral tracing and c-fos immunostaining revealed the involvement of various subcortical areas, especially the EP-lateral habenula (LHb)-ventral tegmental area (VTA) circuit, in SIB. Furthermore, regulating activity in the LHb or the VTA alleviates SIB. These results may have implications in the development of new strategies for treating SIB.

\section{Introduction}

Self-injurious behavior (SIB) often occurs in humans as a repetitive or compulsive action to harm one's own body (Schroeder et

Received Sept. 5, 2017; revised April 4, 2018; accepted April 13, 2018

Author contributions: Y.G., X.T., J.Z., X.H., F.X., G.-Q.B., L.X., and P.-M.L. designed research; Y.G., X.T., J.Z., S.J., J.L., K.Z., and H.Z. performed research; Y.G., X.T., L.D., and C.Y. analyzed data; Y.G., X.T., J.Z., G.-Q.B., L.X., and P.-M.L. wrote the paper. al., 2001). The prevalence of SIB is $\sim 4 \%$ in the general population and $\leq 50 \%$ in patients with neuropsychiatric disorders (Briere and Gil, 1998; Devine, 2014; Bentley et al., 2015). Various risk factors for SIB have been identified, including neurodevelop- 
mental defects, such as those in patients with autism spectrum disorders (ASDs); psychological conditions, such as abuse in childhood; and genetic disorders, such as Lesch-Nyhan syndrome (Anderson and Ernst, 1994; Favaro et al., 2007; Devine, 2014). Previous studies suggest that SIB is associated with dysfunctions in neuromodulatory systems, especially the monoamine systems (Schroeder et al., 2001).

Environmental stress has been indicated as a strong factor affecting the occurrence of SIB (Beckett et al., 2002; Davenport et al., 2008). Increased severity of tissue injury was found after social defeat stress in rats (Muehlmann et al., 2012). Although the underlying mechanisms are unclear, it has been hypothesized that SIB may serve as a means of relieving negative feelings (Klonsky, 2007). On the other hand, clinical studies showed seemingly contradictory results on the change in stress-hormone level in patients with neuropsychiatric disorders and SIB. Increased blood cortisol levels were found in adults with developmental disabilities and chronic self-injury (Symons et al., 2003), whereas no change or decrease in cortisol level was found in individuals with autism (Sandman et al., 1991; Curin et al., 2003). Nonetheless, it is generally believed that SIB is linked to stress-related response, but exactly how it is regulated by stress is still unknown.

It is also known that SIB may involve repetitive and stereotypic behavior, especially in some neuropsychiatric patients (Bodfish, 2007). Abnormal repetitive activity can cause severe tissue damage. Such activity includes self-biting in mental retardation patients or excessive grooming in a mouse model of ASD with altered basal ganglia function (Peça et al., 2011; Muehlmann and Lewis, 2012). Impaired functional activity in basal ganglia structures has also been implicated in SIB. For example, bilateral injection of muscimol into the substantia nigra $(\mathrm{SN})$ of rats could induce stereotyped self-biting behavior (Baumeister and Frye, 1984). Lesions in the globus pallidus of the basal ganglia have been reported in some patients of depression, schizophrenia, and obsessive-compulsive disorder (Laplane et al., 1989), psychiatric conditions with high SIB occurrence (Klonsky, 2007). Notably, deep brain stimulation directed at the internal globus pallidus (GPi) can result in mood changes (Okun et al., 2003) and suicidal behavior (Foncke et al., 2006), implying that this area, commonly known for movement control, may also be involved in emotion modulation.

As SIB is often triggered by negative emotions that lead to stereotypic self-harming movements, it is possible that GPi, which is involved in both movement control and emotion regulation, plays an important role in the process of SIB. It has been shown that the entopeduncular nucleus (EP), the rodent homolog of the GPi, sends reward-related signals to the lateral habenula (LHb; Shabel et al., 2012), which is associated with various mental disorders (Li et al., 2013; Chan et al., 2017). The LHb is an important circuitry hub regulating monoamine nuclei, such as the ventral tegmental area (VTA; Proulx et al., 2014). Both the LHb and the VTA are parts of a complex emotion circuitry involved in responses to stress (Hikosaka, 2010; Holly and Miczek,

We thank Qiaogiong Liu, Yan Lu, Meiyu Shi, Yanqing Yin, and Drs. Wei Wu, Fan Jia, and Huadong Wang for their technical help, and Drs. Gal Richter-Levin and Liping Wang for helpful discussions.

The authors declare no competing financial interests.

*Y.G and X.T. contributed equally to this work.

Correspondence should be addressed to either of the following: Dr. Pak-Ming Lau, School of Life Sciences, University of Science and Technology of China, 443 Huangshan Road, Hefei, Anhui 230027, China, E-mail: plau@ustc.edu.cn; or Dr. Lin Xu, Laboratory of Learning and Memory, Kunming Institute of Zoology, Chinese Academy of Sciences, Kunming, Yunnan 650223, China, E-mail: Ixu@vip.163.com.

DOI:10.1523/JNEUROSCI.2540-17.2018

Copyright $\odot 2018$ the authors $\quad 0270-6474 / 18 / 385252-16 \$ 15.00 / 0$
2016), which is known to affect various brain functions (Xu et al., 1998; Park et al., 2017) and to exacerbate SIB (Devine, 2012).

It is thus conceivable that the above brain circuits play important roles in the induction and regulation of SIB. In the current study, we have established a rat model of SIB by impairing neural activity in the EP. With this model, we were able to systematically examine which brain areas are involved in SIB, and to investigate how this behavior is modulated by stress.

\section{Materials and Methods}

Animals. All animal experiments were conducted following protocols approved by the Animal Experiments Committees at the University of Science and Technology of China, Wuhan Institute of Physics and Mathematics, Kunming Institute of Zoology, the Chinese Academy of Sciences, and Kunming University of Science and Technology. Male adult (8-10 weeks of age) Sprague Dawley rats (Vital River Laboratories or Wuhan Centers for Disease Prevention \& Control) were used for all experiments. The rats were housed under a $12 \mathrm{~h}$ light/dark cycle with ad libitum access to food and water.

Stereotaxic surgery. Rats were anesthetized with pentobarbital sodium salt (60 mg/kg; Merck) and placed in a stereotactic apparatus (RWD Life Science). For drug injections, small holes were drilled in the skull for the implantation of stainless steel guiding cannulae (500 $\mu \mathrm{m}$ outer diameters, $350 \mu \mathrm{m}$ inner diameters) positioned $1.0 \mathrm{~mm}$ above the target brain areas (EP: $2.5 \mathrm{~mm}$ posterior, $3.0 \mathrm{~mm}$ lateral, $6.7 \mathrm{~mm}$ ventral to bregma; LHb: $3.8 \mathrm{~mm}$ posterior, $0.8 \mathrm{~mm}$ lateral, $4.0 \mathrm{~mm}$ ventral to bregma; VTA: $5.8 \mathrm{~mm}$ posterior, $0.7 \mathrm{~mm}$ lateral, $7.4 \mathrm{~mm}$ ventral to bregma). The location of the injection site was examined using Nissl staining. Animals with off-target injections were excluded from further analysis unless otherwise stated.

For viral tracing experiments, pseudorabies virus (PRV) carrying the gene for enhanced green fluorescent protein (PRV-152-EGFP) was injected into the left or right masseter muscle around the marginal mandibular branch $\left(5 \times 10^{9} \mathrm{U} / \mathrm{ml}\right.$, three sites, $1.5 \mu$ l per site; Stanek et al., 2014; Wei et al., 2015). Since it takes $\geq 48 \mathrm{~h}$ for PRV to infect neurons in the brain from muscles (Fay and Norgren, 1997), we injected herpes simplex virus (HSV) carrying the tdTomato gene (HSV-tdTomato) into the EP $\left(5 \times 10^{10} \mathrm{U} / \mathrm{ml}, 300 \mathrm{nl}\right.$; Tang et al., 2016) ipsilateral to the PRV injection site $2 \mathrm{~d}$ later. Animals were then housed in home cages for $60 \mathrm{~h}$ before being killed.

Behavioral experiments and analyses. After surgery, each rat was allowed to recover in its individual home cage for $7 \mathrm{~d}$ before being transferred to a black plastic recording box $(30 \times 30 \times 30 \mathrm{~cm})$ with an infrared transparent floor and two infrared cameras installed above and below the box. The rat was habituated in the recording box for $3 \mathrm{~d}$ ( $8 \mathrm{~h}$ per day) before intracranial injections. For intracranial injection, the rat was anesthetized with isoflurane (3\% for induction and $1.5 \%$ for maintenance; RWD Life Science). For SIB induction, $1.0 \mu \mathrm{l}$ of muscimol (SigmaAldrich) solution of various concentrations was injected bilaterally into the EP using a syringe pump at $0.25 \mu \mathrm{l} / \mathrm{min}$. After injection, the cannula was left in place for an additional minute to allow diffusion of the drug away from the cannula tip. In other experiments, $1.0 \mu \mathrm{l}$ of muscimol (1.0 $\mu \mathrm{g} / \mu \mathrm{l}$ ) or bicuculline methiodide (BMI; $200 \mu \mathrm{M}$; Tocris Bioscience) was injected into target brain areas (e.g., LHb or VTA) bilaterally 20-30 min before SIB induction. RU38486 (RU; $60 \mathrm{mg} / \mathrm{kg}$; Sigma-Aldrich) or corticosterone ( $60 \mathrm{mg} / \mathrm{kg}$; TCI Chemicals) was intraperitoneally injected 15 min before SIB induction. Metyrapone ( $100 \mathrm{mg} / \mathrm{kg}$; Sigma-Aldrich) was intraperitoneally injected for 5 consecutive days; on the fifth day, metyrapone was injected 15 min before SIB induction. For restraint stress experiments, the rat received acute restraint in a restraint box for $3 \mathrm{~h}$ before intracranial injection. Immediately after injection, the animal was transferred to the recording box, allowed 5-10 min to recover from anesthesia, and recorded for $\leq 7 \mathrm{~h}$. After recording, each rat was visually examined and photographed for wounds on different body regions, including forepaws, chest, belly, legs, hindpaws, and tail. Severity of wounds was quantified using criteria similar to those of previous studies (Turner et al., 1999). 
A

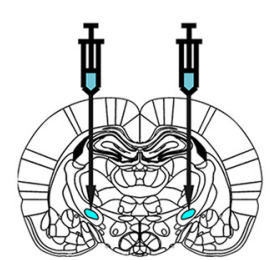

Entopeduncular nucleus (EP)

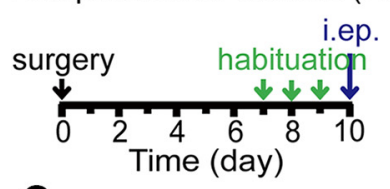

C

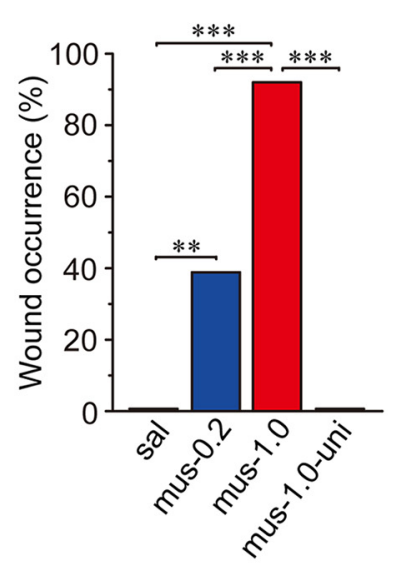

B

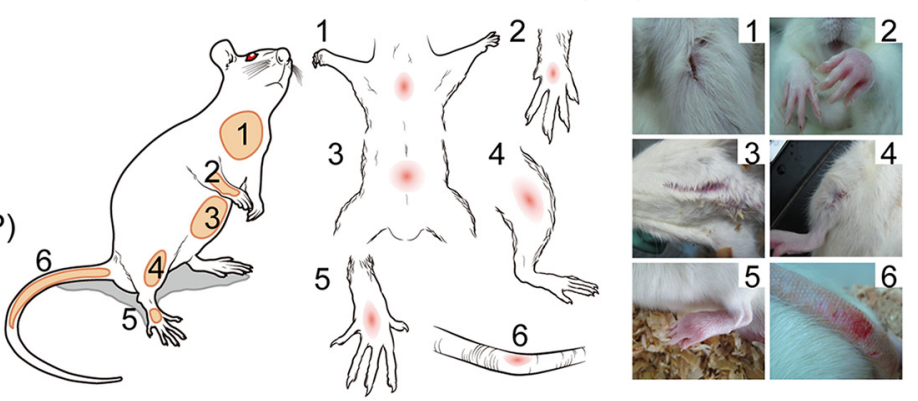

D

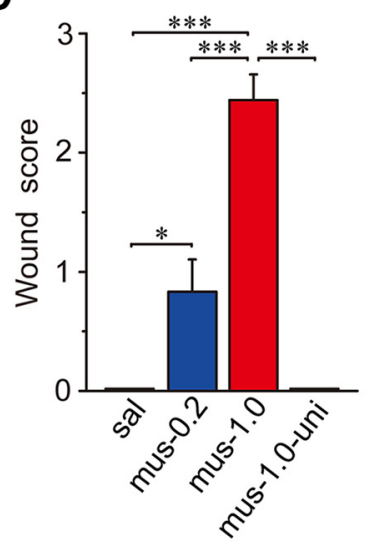

E
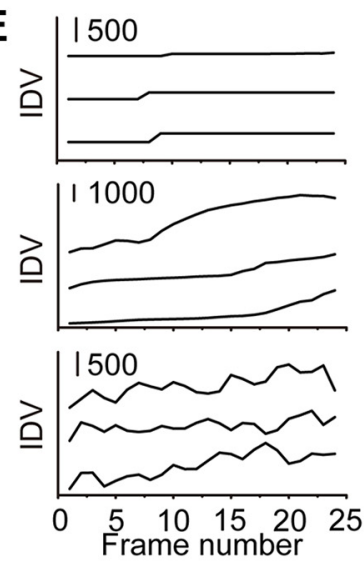

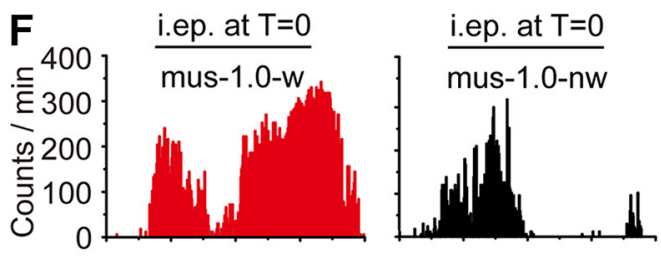

sal

H

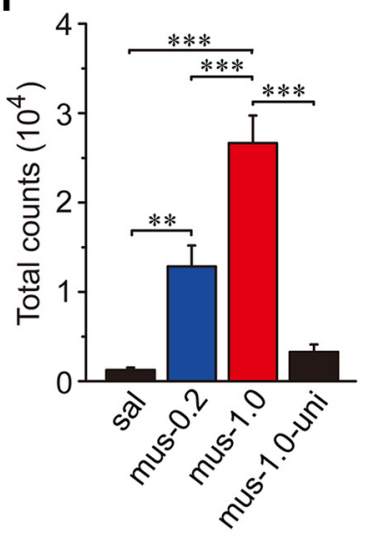

(min)

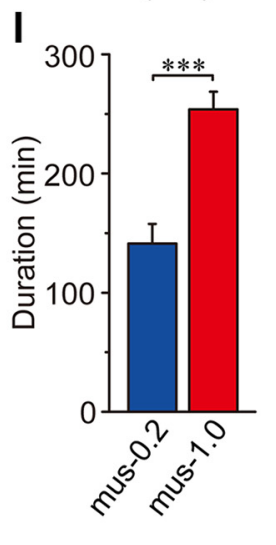

G
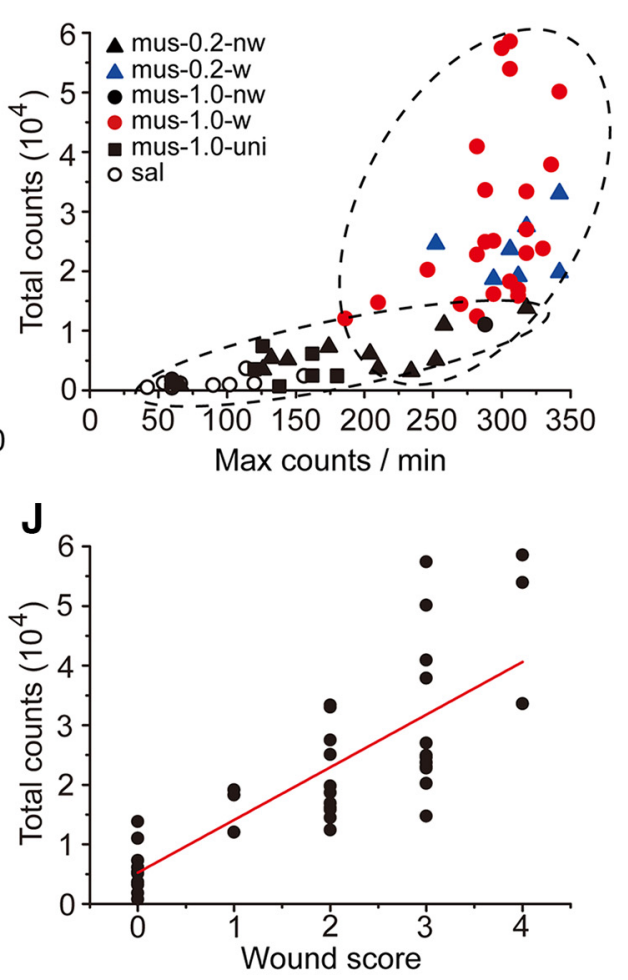

Figure 1. Stereotypic SIB induced by disruption of activity in the EP. A, Experimental paradigm. Saline (sal) or muscimol (mus) was injected into the EP bilaterally. EP injection was performed $10 \mathrm{~d}$ after surgery, following $3 \mathrm{~d}$ of habituation. i.ep., EP injection. B, Schematic diagram and example photos of SIB rats, showing wounds on chest (1), forepaws (2), belly (3), legs (4), hindpaws (5), and tail (6). C, SIB was induced by muscimol injection into the EP, with the wound-occurrence ratio of the mus- 1.0 group significantly higher than that of the mus- 0.2 group. No wound occurred in the saline and unilateral (uni) muscimol injection (mus-1.0-uni) groups. D, Average score of wound severity for each group. $\boldsymbol{E}$, Examples of IDV curves corresponding to different types of animal movement. Three IDV curves, each based on a 1 s video segment when the rat was at rest (top), moving naturally (middle), or moving repetitively (bottom). Scale bars: top and bottom, 500 IDVs; middle, 1000 IDVs. F, Quantification of repetitive movements of four individual animals after EP injections. The movements included self-biting, grooming, and (Figure legend continues.) 
Custom software was developed to quantitatively analyze repetitive movements in rats. The algorithm is based on the analysis of the image difference value (IDV) between two video frames, defined as the sum of the absolute values of all pixel intensities in one frame subtracting those of corresponding pixels in the other frame. The algorithm is expressed as follows:

$$
\mathrm{IDV}=\sum_{i j} H\left(\left|F_{i j}^{A}-F_{i j}^{B}\right|-t\right)
$$

$F_{i j}^{A}$ and $F_{i j}^{B}$ represent pixel matrices of frames $A$ and $\mathrm{B}$, respectively. $H$ is a step function. $H(x)=1$ when $x \geq 0$ and $H(x)=0$ otherwise. A threshold parameter $t$ was chosen (usually $\sim 60$ ), depending on the quality of video images.

For each 1-s-long video segment ( 25 frames per second), a time series of 24 non-negative IDVs was generated by comparing the first frame with each of the remaining 24 frames in the segment. Three different types of animal movement could be classified based on the pattern of the IDV time series (Fig. 1E). When the rat stayed still, all values in the time series were close to zero. When the rat moved naturally, the time series often showed monotonic increase. When the rat made repetitive movements, the time series was near rhythmic, usually $\sim 6 \mathrm{~Hz}$. Such stereotypic movements identified and quantified by the program were confirmed by manual analysis of video clips. Codes and executable programs are available upon request.

Measurement of serum corticosterone. Rats were anesthetized with isoflurane $(5 \%)$ for blood collection via tail vein $(0.2 \mathrm{ml}$ each time, for measuring serum corticosterone changes over time), or by cardiac puncture $(0.3 \mathrm{ml}$ per animal, for acute restraint stress experiments). Serum was extracted and stored at -20 or $-80^{\circ} \mathrm{C}$ until assayed. Corticosterone level was measured using an ELISA kit for corticosterone (Enzo Life Science).

Immunostaining, fluorescence imaging, and image analysis. Rats were killed by an overdose of pentobarbital sodium salt, followed by intracardiac perfusion with $4 \%$ paraformaldehyde (PFA) in phosphate buffer saline. Brains were extracted from the skulls and kept in $4 \%$ PFA at $4{ }^{\circ} \mathrm{C}$ for $24 \mathrm{~h}$. After cryoprotection in $30 \%$ sucrose, coronal slices $(30 \mu \mathrm{m}$ thick for $\mathrm{c}$-fos immunofluorescence staining, and $50 \mu \mathrm{m}$ thick for all other experiments) were immunostained using one primary antibody or a combination of primary antibodies, including rabbit anti-c-fos (1:500; Santa Cruz Biotechnology, catalog \#sc-52, RRID:AB_2106783), mouse anti-tyrosine hydroxylase (TH; 1:1000; Millipore, catalog \#MAB318, RRID:AB_2201528), rabbit anti-DsRED (1:1000; Clontech Laboratories, catalog \#632496, RRID:AB_10013483), and rabbit anti-GFP conjugated with Alexa 647 (1:1000; Invitrogen, catalog \#A31852, RRID:AB_162553). Immunostains were amplified with appropriate secondary antibodies, including Alexa Fluor488-labeled goat anti-rabbit (1:500; Jackson ImmunoResearch Laboratories, catalog \#111-545-144, RRID:AB_2338052), Cy3-labeled donkey anti-mouse (1:500; Jackson ImmunoResearch Laboratories, catalog \#715-165-151, RRID:AB_2315777), and Cy3-labeled goat anti-rabbit (1:500; Jackson ImmunoResearch Laboratories, catalog \#111-165-144, RRID:AB_2338006). Fully stained samples were imaged using a fluorescence stereomicroscope (Olympus, MVX10) with a $2 \times$

\footnotetext{
(Figure legend continued.) head nodding. G, Total counts of movements in 400 min after EP injection versus the maximal counts of movements per minute for different groups of animals. Color symbols indicate rats with wounds; black symbols indicate rats with no wounds. $\boldsymbol{H}$, The total counts of movements over the 400 min of the mus- 1.0 group were significantly more than those of the mus- 0.2 group, mus-1.0-uni group, and the saline group. I, Duration of the repetitive movements of the mus- 1.0 group was significantly longer than that of the mus- 0.2 group. $J$, Correlation of total repetitive movements and wound-severity score. Each point indicates a muscimol-injected rat. The red line represents the best fit with linear regression between the repetitive movements and the wound score. ${ }^{*} p<0.05,{ }^{* *} p<0.01,{ }^{* * *} p<0.001$ for all figures. mus- 0.2 , Rats bilaterally injected with $0.2 \mu \mathrm{g}$ of muscimol per site; mus-1.0, rats bilaterally injected with $1.0 \mu \mathrm{g}$ of muscimol per site; mus-1.0-uni, rats unilaterally injected with $1.0 \mu \mathrm{g}$ of muscimol in one site and saline in the other; sal, rats bilaterally injected with saline; $\mathrm{w}$, rats with wounds; $n \mathrm{nw}$, rats without wounds. Number of animals used: sal, $n=12$; mus- $0.2, n=18$; mus-1.0, $n=25$; mus-1.0-uni, $n=8$.
}

Table 1. Severity of SIB

\begin{tabular}{|c|c|c|}
\hline Score & Severity & Description \\
\hline 0 & No injury & No tissue damage \\
\hline 1 & Very mild injury & $\begin{array}{l}\text { Slight edema, pink moist skin, denuded skin }\left(<20 \mathrm{~mm}^{2}\right) \text {, involves } \\
\text { multiple sites }\end{array}$ \\
\hline 2 & Mild injury & $\begin{array}{l}\text { Obvious edema, obvious erythema, denuded skin involves large } \\
\text { area }\left(\geq 20 \mathrm{~mm}^{2}\right) \text {, skin damage involves medium area } \\
\left(<10 \mathrm{~mm}^{2}\right) \text {, involves multiple sites }\end{array}$ \\
\hline 3 & Moderate injury & $\begin{array}{l}\text { Substantial skin damage involves large area }\left(\geq 10 \mathrm{~mm}^{2}\right) \text {, tissue } \\
\text { loss }\end{array}$ \\
\hline 4 & Severe injury & Amputation of digit, clear lesions, requires animal to be killed \\
\hline
\end{tabular}

objective (numerical aperture, 0.5 ), at $2-4 \times$ zoom. TH and c-fos confocal images were captured on a confocal microscope (Zeiss, LSM710) with a $20 \times$ water-immersion objective (numerical aperture, 1.0).

Semiquantitative analyses of c-fos expression and viral tracing signals were performed for all brain areas by visual inspection. For each given area, the c-fos expression was considered substantially increased if the number of c-fos-positive neurons in the rats examined $4 \mathrm{~h}$ after injection of muscimol ( $1.0 \mu \mathrm{g} / \mu \mathrm{l}$; mus-1.0-4 h group) was $>30$ and was $\geq 3$ times the number of c-fos-positive neurons in rats treated similarly but with saline (sal- $4 \mathrm{~h}$ group). For viral tracing data, a brain area was considered to have strong coinfection of HSV and PRV if the density of both types of infected neurons was $>40$ per square millimeter.

Quantitative analysis of c-fos expression in selected brain areas was performed using a custom Python program. After image smoothing, c-fos-positive cells were identified by visual inspection, with coordinates of all identified cells marked manually. The program then measured the signal (defined as the maximal pixel intensity within a $14 \times 14 \mu \mathrm{m}^{2}$ patch surrounding an artificial mark) and the background (defined as the median intensity of the pixels on the border of a $42 \times 42 \mu \mathrm{m}^{2}$ patch surrounding the peak intensity point) of the cell. Only cells with the signal/noise ratio $>1.2$ were counted as true c-fos-positive cells. This procedure eliminates human biases influenced by different imaging conditions.

Experimental design and statistical tests. For SIB induction/glucocorticoid modulation/restraint/c-fos expression/viral tracing/LHb-VTA modulation experiment, 63/145/79/28/4/95 rats with correct injection loci were used. For the systematic survey of whole-brain c-fos or viral tracing signal, six consecutive sections were examined for each brain area, and the section with the highest density of positive neurons was selected for semiquantitative analysis. For quantitative analysis of c-fos expression in selected brain areas, the number of c-fos-positive cells on six sections was counted for each area (three consecutive sections from each hemisphere; except for the VTA, where three consecutive sections were counted as both hemispheres can be counted together in the same brain section). The wound-occurrence ratio for each group was determined as the ratio of the number of rats with wounds to the total number of rats. Changes in corticosterone following bilateral muscimol or saline injection were compared using two-way repeated-measures ANOVA for determination of main effects and interactions, followed by least significant difference (LSD) post hoc tests for pairwise comparisons among groups. Data of c-fos-positive cell counting, except for those from unilateral muscimol-injection experiments, were analyzed using two-way ANOVA followed by LSD post hoc tests to compare the effect of different drugs at different times. All other data were analyzed using either oneway ANOVA followed by LSD post hoc tests (for tests with $>2$ groups) or by two-sample Student's $t$ test (for tests with two groups), and expressed as mean \pm SEM. Test results were considered statistically significant when $p<0.05$.

\section{Results}

SIB in rats induced by muscimol injection in the EP

After habituation in the recording box for $3 \mathrm{~d}$, rats were injected bilaterally with muscimol into the EP to disrupt local neural activity (Fig. 1A). Immediately after injection, the rats exhibited agitated movements for about $10 \mathrm{~min}$, and then gradually quieted 
A

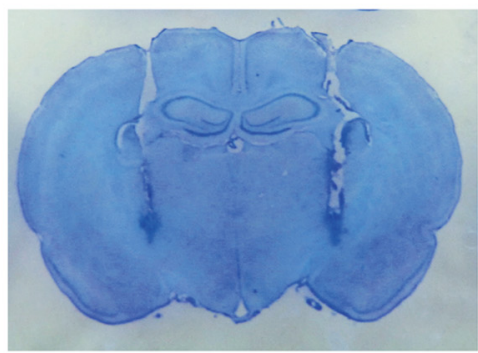

B

sal

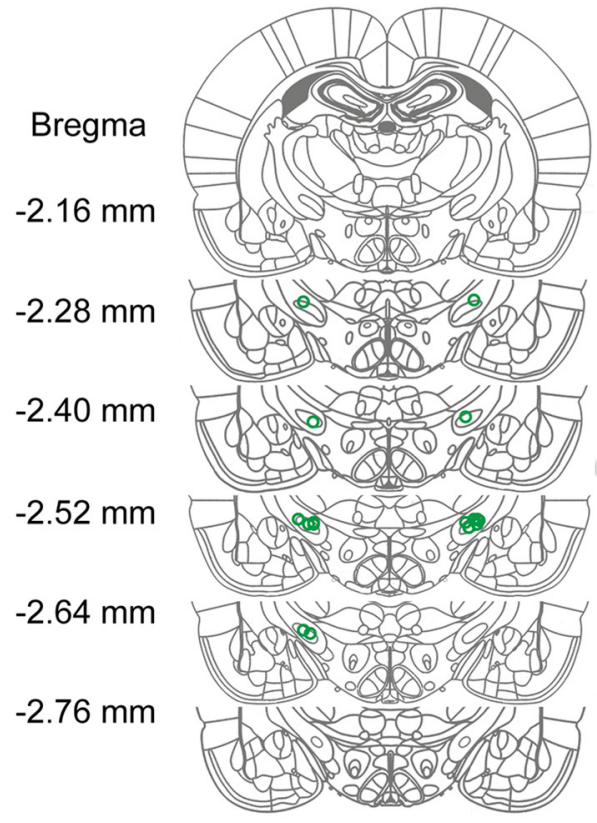

mus-1.0

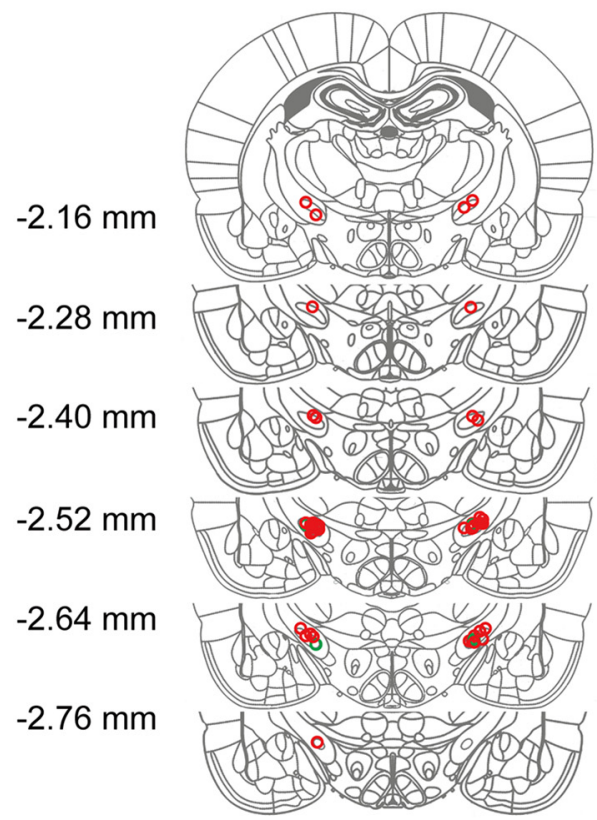

o no wound o wounds

mus-0.2

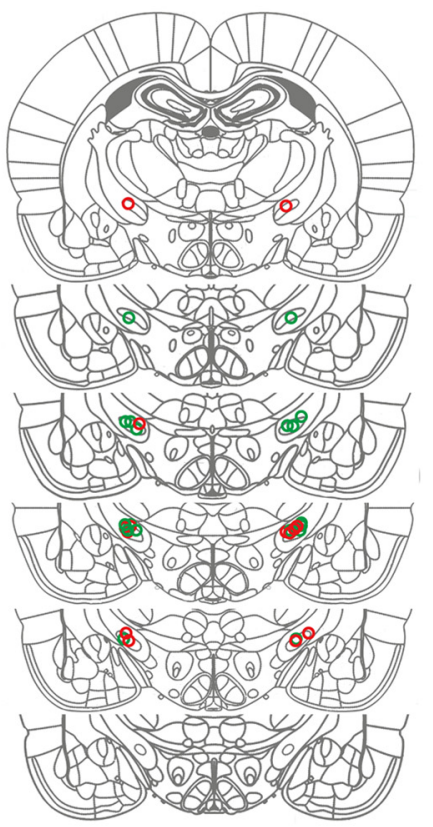

mus-1.0-uni

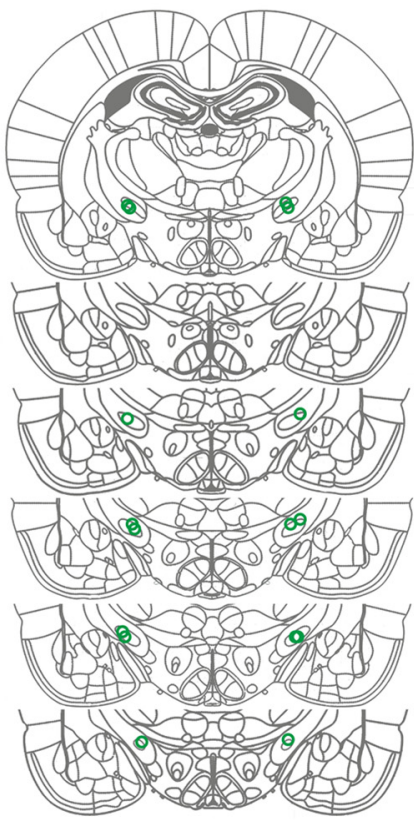

Figure 2. EP injection sites in all the animals used in Figure 1. $A$, An example brain slice with Nissl staining showing cannulae locations in the EP. B, Summary of verified injection sites shown on a brain atlas (red, rats with wounds; green, rats with no wounds).

down as their limb muscles started to show stiffness and stopped locomotor activity. Approximately 40-60 min following the muscimol injection, most rats curled up and began to show repetitive movements, including stereotypic sniffing, head nodding, and self-biting. Such stereotypic self-biting behaviors were always accompanied by other stereotypic movements and lasted 2-5 h, which usually led to skin and tissue damage in one or more body areas of the rats (Fig. $1 B$ ). We considered this behavior a form of SIB and called these animals with wounds "SIB rats." For the group with bilateral injection of $1.0 \mu \mathrm{g}$ of muscimol on each side (mus-1.0), wounds occurred in about $90 \%$ of the rats. The wound-occurrence ratio fell to $\sim 40 \%$ in the low-dose group (mus-0.2), whereas no wounds were found in the control groups with bilateral saline (sal) injection or unilateral (uni) muscimol injection $\left(F_{(3,59)}=24.827, p<0.001\right.$; sal vs mus-0.2: $p=0.003$; sal vs mus-1.0: $p<0.001$; mus-0.2 vs mus-1.0: $p<0.001$; mus-1.0-uni vs mus-1.0: $p<0.001$; Fig. $1 C)$. Wound severity was also scored for each group of rats $\left(F_{(3,59)}=27.042, p<\right.$ 0.001 ; sal vs mus-0.2: $p=0.019$; sal vs mus-1.0: $p<0.001$; mus-0.2 vs mus-1.0: $p<0.001$; mus-1.0-uni vs mus-1.0: $p<$ 0.001; Fig. $1 D$, Table 1).

We further analyzed and quantified the repetitive movements in rats using custom software (see Materials and Methods; Fig. $1 E$ ). The SIB animals in general showed more repetitive movements and higher repetition frequency than the nonSIB (those with stereotypic movements but no wounds) or the control rats $\left(F_{(3,59)}=\right.$ 18.093, $p<0.001$; sal vs mus-0.2: $p=$ 0.007; sal vs mus-1.0: $p<0.001$; mus-0.2 vs mus-1.0: $p<0.001$; mus-1.0-uni vs mus-1.0: $p<0.001$; Fig. $1 F-H$ ), as well as longer duration of repetitive movements [mus-0.2 vs mus-1.0, $t_{(41)}=5.013, p<$ 0.001; Fig. 1I]. Notably, rats with unilateral muscimol injection exhibited moderate head movements but no stereotypic selfbiting behavior, whereas rats with saline injection showed normal behavior with occasional grooming (Fig. $1 F$ ). A positive correlation was found between the score of wound severity and the counts of total movements in the bilateral muscimolinjection groups $\left(R^{2}=0.64\right.$; Fig. $\left.1 J\right)$. The $\mathrm{EP}$ injection sites in all the animals used in Figure 1 were mapped out (Fig. 2). These results suggest that the occurrence of SIB is linked to the level of stereotypic movements in a dose-dependent manner.

To determine whether EP is indeed the induction site of such SIB, we mapped out 
the muscimol-injection loci of all SIB and non-SIB rats, and further included cases of deliberately deviated injection about $0.8-1.0 \mathrm{~mm}$ away from the edge of the EP in the dorsal-ventral or medial-lateral direction (Fig. 3A). Along the rostral-caudal axis, the wound-occurrence ratio in the mus- 0.2 group was relatively higher at loci closer to the target location of our $\mathrm{EP}$ injection site $(2.50 \mathrm{~mm}$ posterior to bregma; Fig. 3B). We further analyzed the wound-occurrence ratio around the EP area near the target injection site (from 2.40 to $2.64 \mathrm{~mm}$ posterior to bregma) and found that the ratio decreased rapidly as the injection sites deviated from the EP (Fig. 3D). A similar trend was observed in the mus-1.0 group (Fig. 3C,E). These results suggest that the disruption of neural activity in the EP rather than its adjacent areas is responsible for the stereotypic movements and subsequent self-injury in rats.

\section{Modulation of SIB by}

\section{glucocorticoid signaling}

Previous studies have shown that SIB is accompanied by changes in the level of stress hormone (Symons et al., 2003). We measured the serum levels of corticosterone (the main glucocorticoid in rodents) in rats following muscimol injection at the EP. As shown in Figure $4 A$, the corticosterone levels in the muscimol-injected groups (mus-1.0 and mus-0.2) increased rapidly and were significantly higher than the control group (sal) $1 \mathrm{~h}$ after the injection. In the mus-1.0 group, corticosterone kept rising steadily and reached the maximal level about $4 \mathrm{~h}$ after injection. After $4 \mathrm{~h}$, the level started to fall. In the mus- 0.2 group, the corticosterone level began to fall slightly 2-4 h after injection, but was still significantly higher than that in the control group, before decaying to baseline level by $4-6 \mathrm{~h}$. In contrast, corticosterone in the control group remained at a low level and only rose moderately in the afternoon due to the normal daily cycle of the hormone. Two-way repeated-measures ANOVA of this dataset showed a significant effect of drug injection $\left(F_{(2,24)}=\right.$ $19.164, p<0.001)$, time $\left(F_{(2.909,69.825)}=\right.$ $20.490, p<0.001)$, and drug $\times$ time interaction $\left(F_{(5.819,69.825)}=11.643, p<\right.$ $0.001)$. LSD post hoc tests showed a significant difference of drug injection among the three groups (sal vs mus- $0.2, p=$ 0.018 ; sal vs mus-1.0, $p<0.001$; mus- 0.2 vs mus-1.0, $p<0.001$; Fig. $4 A$ ). All eight rats in the mus-1.0 group and five of 11 rats in the mus- 0.2 group exhibited biting wounds. We further compared corticoste-
A
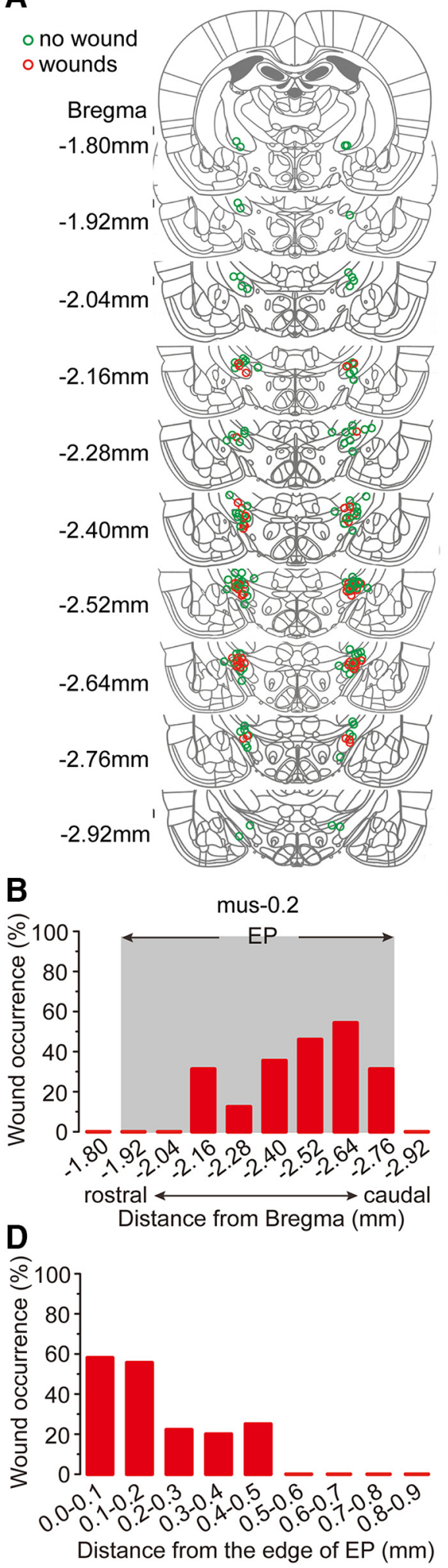
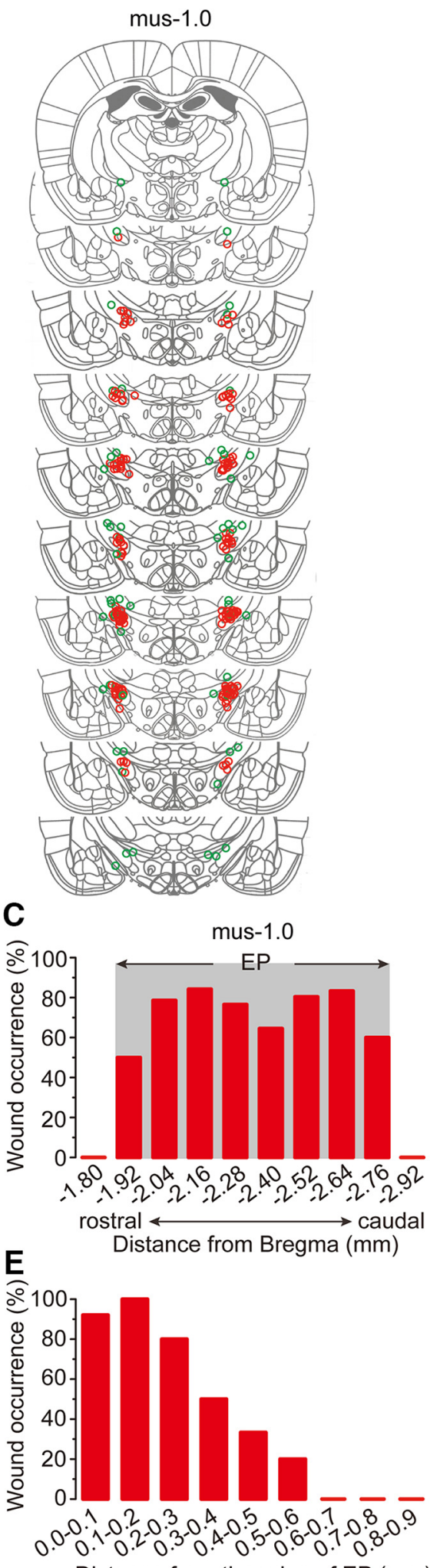

Figure 3. Occurrence of SIB sharply decreased as the muscimol injection sites deviated from the EP. $\boldsymbol{A}$, Summary of EP injection sites in all SIB and non-SIB rats shown on a brain atlas (red, rats with wounds; green, rats with no wounds). $\boldsymbol{B}$, Wound ratio along the sagittal axis in the mus- 0.2 group. The gray zone indicates the range of the EP. $\boldsymbol{C}$, Wound ratio along the sagittal axis in the mus-1.0 group. $\boldsymbol{D}$, Wound ratio versus the distance from the edge of the EP to the center of injection sites in the mus-0.2 group (from 2.40 to $2.64 \mathrm{~mm}$ posterior to bregma). $\boldsymbol{E}$, Wound ratio versus the distance from the edge of the EP to the center of injection sites in the mus-1.0 group (from 2.40 to $2.64 \mathrm{~mm}$ posterior to bregma). 

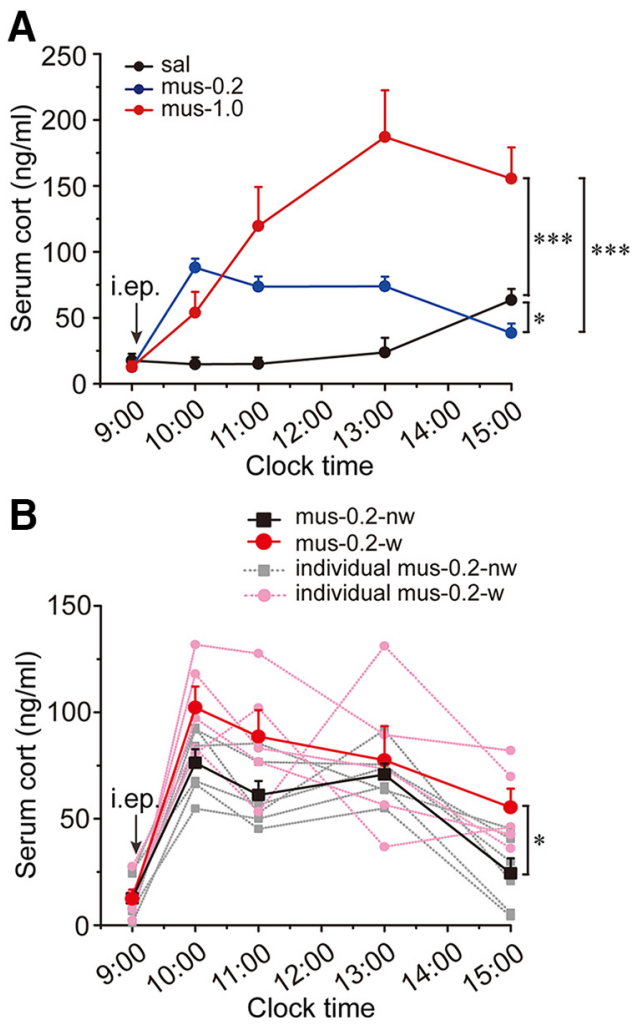

Figure 4. The elevated corticosterone level was associated with the induction and maintenance of SIB. $A$, The corticosterone levels in the mus- 0.2 and mus- 1.0 groups increased rapidly following the muscimol injection into the EP and remained significantly higher than that in the control group in the $4-6 \mathrm{~h}$ after the injection (sal, $n=8 ;$ mus- $-0.2, n=11$; mus-1.0, $n=8$ ). $B$, Corticosterone levels in the SIB rats were significantly higher than those in the non-SIB rats. The dashed lines represent the corticosterone levels for each individual animal, and the black and red solid lines show the average corticosterone levels for the mus- $0.2-n w$ and mus $-0.2-w$ groups, respectively. w, Rats with wounds; nw, rats without wounds. ${ }^{*} p<0.05,{ }^{* * *} p<0.001$ for all figures.

rone levels in rats (with and without SIB) of the mus- 0.2 group and found that there was a significant effect of $\operatorname{SIB}\left(F_{(1,9)}=6.605\right.$, $p=0.030)$ and time $\left(F_{(2.055,18.494)}=33.929, p<0.001\right)$, but no significant effect of SIB $\times$ time interaction $\left(F_{(2.055,18.494)}=1.687\right.$, $p=0.212$ ), indicating that corticosterone levels in SIB rats were significantly higher than those in non-SIB rats (Fig. $4 B$ ). These results demonstrate that elevated corticosterone levels are associated with the induction and maintenance of SIB.

To examine whether changes in stress hormone might modulate SIB, we altered the levels of corticosterone in muscimol-injected rats using pharmacological and behavioral manipulations. In the group intraperitoneally injected with glucocorticoid receptor blocker RU38486 (RU; 60 mg/kg) or steroid $\beta$-hydroxylase inhibitor metyrapone (Met; $100 \mathrm{mg} / \mathrm{kg}$ ), animals showed a significant reduction in the wound-occurrence ratio $\left(F_{(10,107)}=15.911\right.$, $p<0.001$; Veh + mus-1.0 vs RU + mus-1.0: $p<0.001 ;$ Veh2 + mus-1.0 vs Met + mus-1.0: $p=0.001$; Fig. $5 A)$ and severity $\left(F_{(10,107)}=14.116, p<0.001 ;\right.$ Veh + mus-1.0 vs RU + mus-1.0: $p<0.001$; Veh2 + mus-1.0 vs Met + mus-1.0: $p=0.005$; Fig. $5 B)$, as well as the total counts $\left(F_{(10,107)}=11.270, p<0.001 ;\right.$ Veh + mus-1.0 vs RU + mus-1.0: $p=0.001$; Veh2 + mus- 1.0 vs Met + mus-1.0: $p=0.030$; Fig. $5 C-E)$ and duration $\left(F_{(6,73)}=12.079\right.$, $p<0.001$; Veh + mus-1.0 vs RU + mus-1.0: $p=0.004 ; \mathrm{Veh} 2+$ mus-1.0 vs Met + mus-1.0: $p=0.016$; Fig. $5 F$ ) of the stereotypic movements compared with rats in the control groups. To elevate the levels of corticosterone, we injected corticosterone (Cort; 60 $\mathrm{mg} / \mathrm{kg}$, i.p.) in the lower-dose group (mus-0.2) to avoid the ceiling effect. The rats exhibited a significantly higher woundoccurrence ratio $\left(F_{(10,107)}=15.911, p<0.001\right.$; Veh + mus- 0.2 vs Cort + mus-0.2: $p=0.001$; Fig. $5 A)$ and severity $\left(F_{(10,107)}=\right.$ 14.116, $p<0.001$; Veh + mus- 0.2 vs Cort + mus-0.2: $p=0.012$; Fig. $5 B$ ) with more severe stereotypic movements compared with the control group (total counts: $F_{(10,107)}=11.270, p<0.001$; Veh + mus- 0.2 vs Cort + mus- $0.2: p=0.002$; duration: $F_{(6,73)}=$ 12.079, $p<0.001$; Veh + mus-0.2 vs Cort + mus-0.2: $p=0.005$; Fig. $5 C-F)$. No SIB occurred in the control groups of rats injected with saline in the EP plus intraperitoneal injection of RU38486, metyrapone, or corticosterone (Fig. 5A,B).

To evaluate the effects of physiological stress on SIB, rats were exposed to acute restraint stress, which is known to activate the hypothalamic-pituitary-adrenal axis and to elevate the systemwide glucocorticoid level (Kirby et al., 2013). Three hours of restraint led to significant enhancement of both neuronal activity in the paraventricular nucleus of hypothalamus (PVN), as reflected by the increase in c-fos expression $\left(t_{(34)}=12.978, p<\right.$ 0.001; Fig. $6 A, B)$, and serum corticosterone $\left(t_{(23)}=4.178, p<\right.$ 0.001 ; Fig. $6 C$ ). Injection of $0.2 \mu \mathrm{g}$ of muscimol (mus-0.2) into the EP right after restraint resulted in significant increase in the wound-occurrence ratio $\left[F_{(4,49)}=16.734, p<0.001\right.$; no restraint + i.p.(mus-0.2) vs restraint + i.p.(mus-0.2): $p=0.003$; restraint + i.p.(sal) vs restraint + i.p.(mus-0.2): $p<0.001$; Fig. $6 D$ ] and severity $\left[F_{(4,49)}=12.973, p<0.001\right.$; no restraint + i.p.(mus- 0.2$)$ vs restraint + i.p.(mus-0.2): $p=0.018$; restraint + i.p.(sal) vs restraint + i.p.(mus-0.2): $p<0.001$; Fig. $6 E$ ] compared with the nonrestraint group. In additional controls, we found no SIB in saline-injected or naive animals with the same stereotaxic surgery (Fig. 6D,E). These results indicate that SIB is modulated by stress, worsened with elevation of corticosterone and ameliorated with reduction of corticosterone.

\section{SIB-related brain areas revealed by activity and connectivity mapping}

To identify the brain areas involved in the process of SIB, we first compared the neuronal activation in the muscimol-injected rats with that in the saline-injected ones using c-fos immunofluorescence staining. The levels of c-fos signal were examined at 1, 2, and $4 \mathrm{~h}$ after injection, which reflected neuronal activities $\sim 1 \mathrm{~h}$ before each time point (Krukoff, 1999). Thus, the c-fos expression in the $1 \mathrm{~h}$ groups reflected the neural activity associated with the handling and immediate response to the injection, whereas the mus-1.0-2 $\mathrm{h}$ and the mus-1.0-4 h groups reflected the activities during the initial and sustained phases of the stereotypic movements, respectively. A systematic semiquantitative analysis of all brain areas (see Materials and Methods) revealed that c-fos signals were substantially enhanced in 36 areas in the mus-1.0-4 h group compared with the saline controls (Fig. 7A).

Because SIB in our rat model was initially induced by disrupting neural activity in the EP, and was eventually expressed as stereotypic self-biting involving contractions of masseter muscle, it is likely that this behavior involves brain areas along the multisynaptic neural signaling pathway from the EP to the masseter muscle. Comparing the brain areas located along this EP-masseter muscle pathway with the above 36 activated brain areas identified by c-fos expression could help narrow down key circuits involved in SIB. To trace the EP-masseter muscle pathway, retrograde PRV carrying the EGFP gene (PRV-EGFP) was injected into the masseter muscle, and anterograde herpes virus expressing tdTomato (HSV-tdTomato) was injected into the EP (Zemanick et al., 1991; Dum and Strick, 2013; Fig. 7B; see Materials and 
A
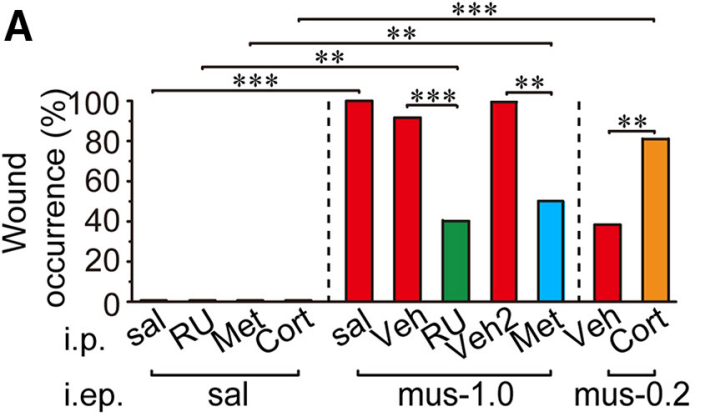

B



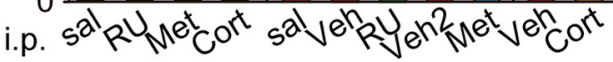

C

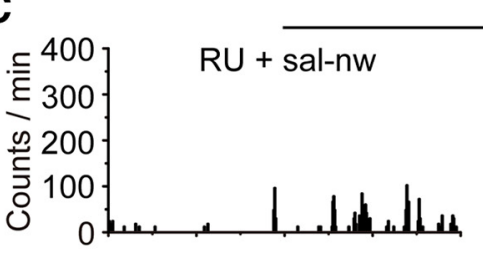

i.ep. at $\mathrm{T}=0$

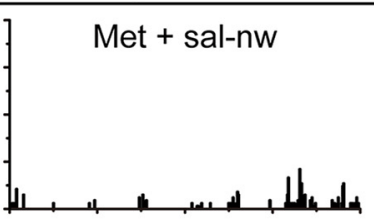

mus-1.0 mus-0.2
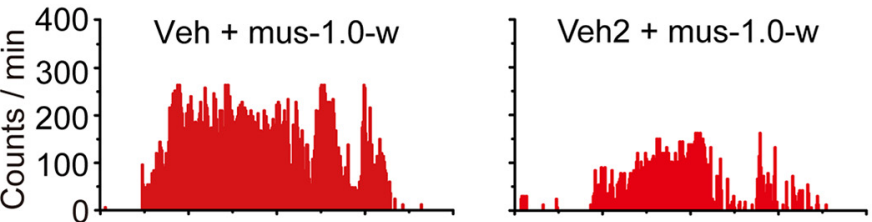

Veh + mus-0.2-nw
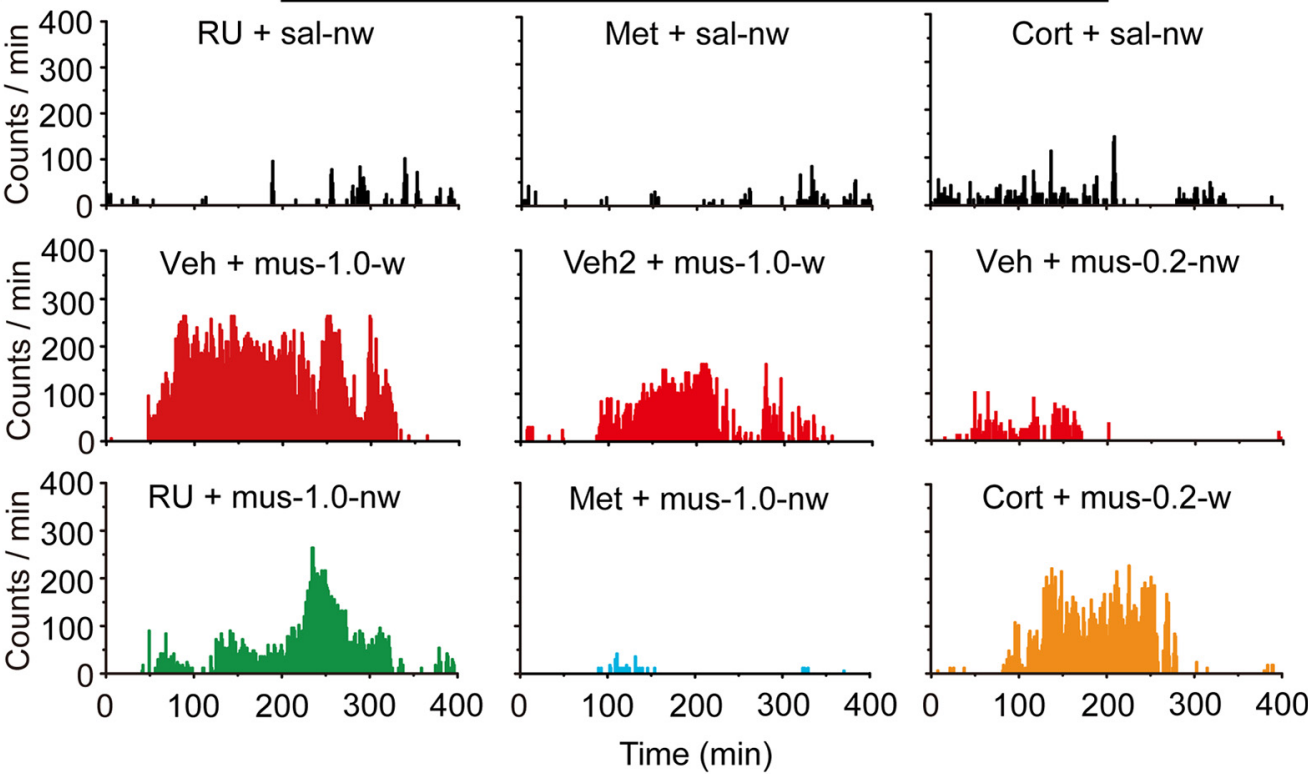

D

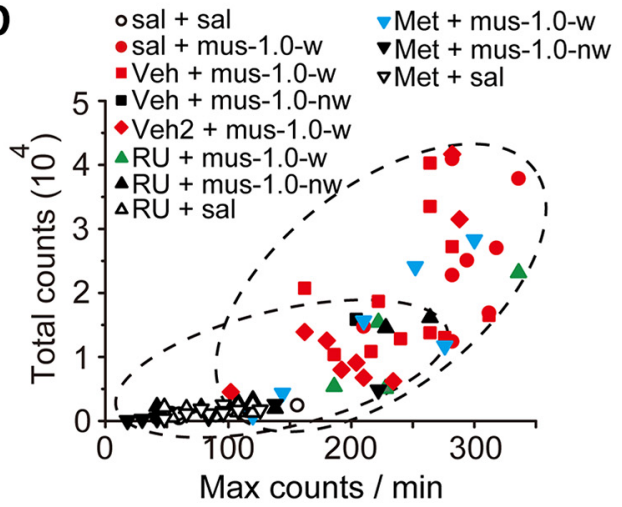

$\mathbf{E}$

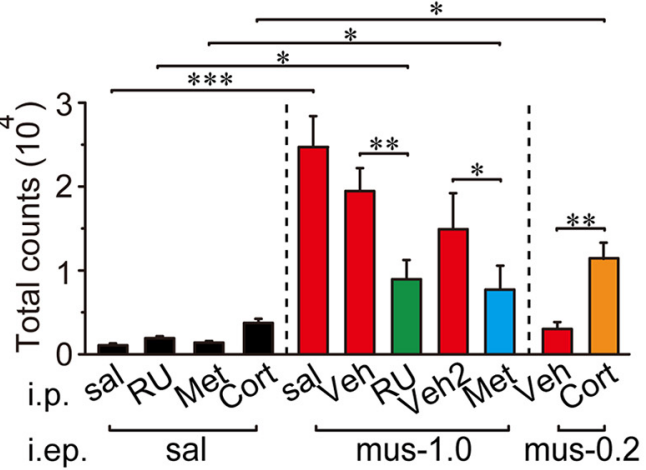

$\mathbf{F}$

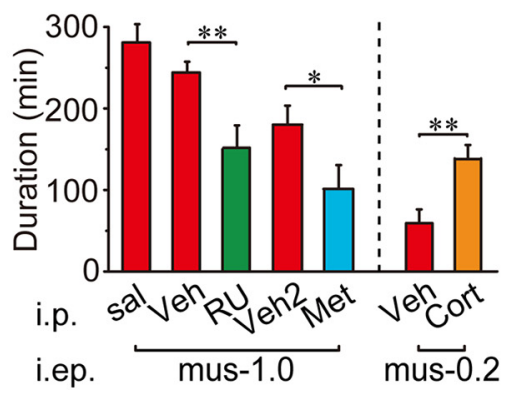

Figure 5. Modulation of SIB by corticosterone level. $A, B$, Intraperitoneal injection of the glucocorticoid receptor antagonist RU38486 (RU) or metyrapone (Met) for 5 consecutive days before EP injection in the mus-1.0 group significantly decreased the wound-occurrence ratio $(\boldsymbol{A})$ and the wound severity $(\boldsymbol{B})$, whereas intraperitoneal injection of corticosterone (Cort) before EP injection in the mus-0.2 group significantly increased the wound-occurrence ratio $(\boldsymbol{A})$ and the wound severity $(\boldsymbol{B} ;$ sal $+\mathrm{sal}, n=8 ; \mathrm{RU}+\mathrm{sal}, n=10 ;$ Met + sal, $n=10$; Cort + sal, (Figure legend continues.) 
A
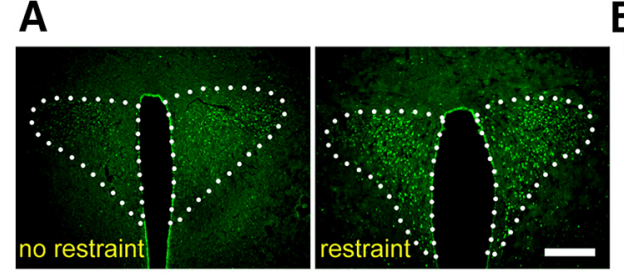

B
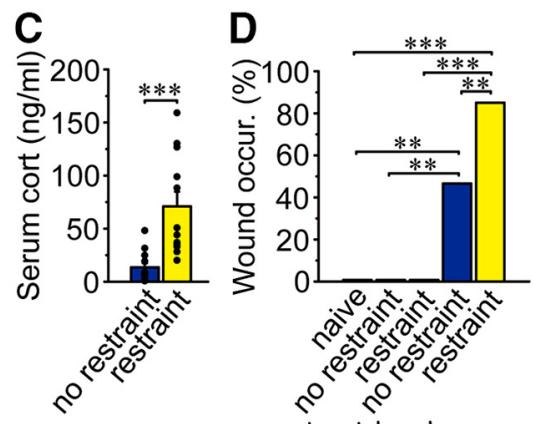

i.ep. sal mus-0.2
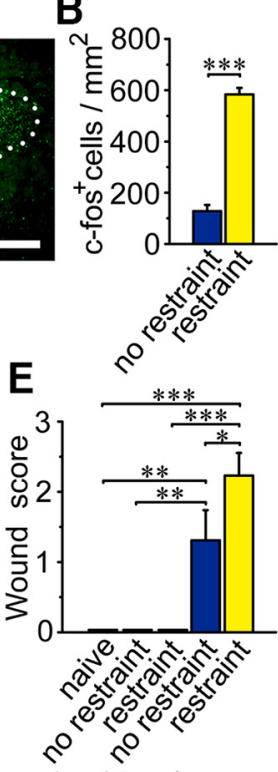

i.ep. sal mus-0.2

Figure 6. Acute restraint stress before EP injection significantly enhanced both neuronal activity in the PVN and serum level of corticosterone, resulted in the increase of woundoccurrence ratio and wound severity. $\boldsymbol{A}$, Representative $c$-fos expression in the PVN of a restraint and a no-restraint control rat. Scale bar, $300 \mu \mathrm{m}$. $\boldsymbol{B}$, The density of c-fos-positive cells in the PVN ( $n=3$ animals for each group, 6 slices for each animal). C, Serum corticosterone significantly increased after $3 \mathrm{~h}$ restraint. The black dot indicates the serum corticosterone level of each individual rat (no restraint, $n=13$; restraint, $n=12$ ). $\boldsymbol{D}, \boldsymbol{E}$, Acute restraint stress before muscimol injection into the EP significantly increased the wound-occurrence ratio $(\boldsymbol{D})$ and the wound severity $[\boldsymbol{E}$; naive, $n=9$; no restraint + i.ep.(sal), $n=9$; restraint + i.ep.(sal), $n=10$; no restraint + i.ep.(mus- 0.2 ), $n=13$; restraint + i.ep.(mus- 0.2$), n=13$ ]. ${ }^{*} p<0.05,{ }^{* *} p<$ $0.01,{ }^{* *} p<0.001$ for all figures.

Methods). Sixty hours after infection, the two viruses could have crossed a few synapses from the two ends of the EP-masseter muscle pathways. We identified 59 brain areas infected by $\geq 1$ type of viral tracers, and 18 of them exhibited relatively strong coinfection of both viruses (Fig. $7 A$ ). In addition, seven brain areas showed both strong coinfection of two viruses and considerable increase of c-fos expression in the mus-1.0-4 $\mathrm{h}$ group. These brain areas were the PVN, LHb, central amygdala (CeA), locus coeruleus (LC), subthalamic nucleus, pedunculopontine tegmental nucleus, and dorsomedial hypothalamic nucleus (Fig. $7 A, C)$.

From the seven identified areas on the EP-masseter muscle pathways and activated during SIB, we first chose two brain areas for further quantitative analysis: the PVN, which is activated by stress and plays a central role in corticosterone release (Herman and Cullinan, 1997), and the LHb, which is a direct target of the

(Figure legend continued.) $\quad n=10 ;$ sal + mus-1.0, $n=8$; Veh + mus-1.0, $n=12 ; \mathrm{RU}+$ mus-1.0, $n=10 ;$ Veh2 + mus-1.0, $n=9$; Met + mus-1.0, $n=12$; Veh + mus- $0.2, n=13$; Cort + mus- $0.2, n=16$ ). C, Quantification of repetitive movements of nine individual animals after intraperitoneal injection of vehicles, RU38486, metyrapone, or corticosterone with EP injection. $\boldsymbol{D}$, Total counts of movements in 400 min after EP injection versus the maximal counts of movements per minute for different groups of animals. Color symbols indicate rats with wounds, while black symbols indicate rats with no wounds. $\boldsymbol{E}, \boldsymbol{F}$, The total counts $(\boldsymbol{E})$ and duration $(\boldsymbol{F})$ of repetitive movements of the RU-injected or Met-injected group were significantly lower, whereas those of the Cort-injected group were significantly higher than the vehicle control groups. Veh, 98\% polyethylene glycol 400 and 2\% ethanol (vehicle); Veh2, propylene glycol (vehicle2). ${ }^{*} p<0.05,{ }^{* *} p<0.01,{ }^{* *} p<0.001$ for all figures.

EP (Shabel et al., 2012) and is also an important player in stress response (Hikosaka, 2010). Indeed, the PVN showed nearly threefold more activated neurons in the mus-1.0-4 h groups as compared with the sal- 4 h groups (drug: $F_{(1,144)}=28.562, p<$ 0.001; time: $F_{(2,144)}=10.779, p<0.001$; drug $\times$ time interaction: $F_{(2,144)}=43.981, p<0.001$; sal vs mus- $1.0,1 \mathrm{~h}: p=0.007 ; 2 \mathrm{~h}: p=$ 0.276; 4 h: $p<0.001$; Fig. $8 A, C$ ), which is consistent with the observed role of stress and corticosterone in SIB (Fig. 6). Furthermore, the LHb showed even more dramatic change, with $>10$ fold higher neuronal activation in the mus-1.0-4 h groups than the sal-4 h control groups (drug: $F_{(1,132)}=201.404, p<0.001$; time: $F_{(2,132)}=3.333, p=0.039$; drug $\times$ time interaction: $F_{(2,132)}=$ 24.133, $p<0.001$; sal vs mus- $1.0,1 \mathrm{~h}: p=0.002$; $2 \mathrm{~h}: p<0.001$; 4 h: $p<0.001$; Fig. $8 B, D$ ), suggesting that the activation of LHb neurons may be involved in SIB. As an additional control, we also examined the c-fos expression $4 \mathrm{~h}$ after unilateral muscimol injection into the EP of one hemisphere with saline injection to the other, a manipulation that did not lead to biting wounds (Fig. $1 C, D)$. Both sides of the PVN [ipsilateral (ipsi) and contralateral (contra)] showed similar elevated c-fos expression, but significantly lower than that of the mus-1.0-4 h group $\left(F_{(3,59)}=18.198\right.$, $p<0.001$; ipsi vs contra: $p=0.822$; mus-1.0-4 h vs ipsi: $p=$ 0.013 ; sal-4 h vs contra: $p<0.001$; Fig. $8 A, C)$. Meanwhile, the ipsilateral $\mathrm{LHb}$ exhibited higher $\mathrm{c}$-fos expression than the contralateral LHb, but this expression was still significantly lower than that in the mus-1.0-4 h group $\left(F_{(3,74)}=81.872, p<0.001\right.$; ipsi vs contra: $p<0.001$; mus-1.0-4 h vs ipsi: $p<0.001$; sal- 4 h vs contra: $p=0.297$; Fig. $8 B, D$ ), supporting a role of LHb activation in SIB.

\section{Modulation of SIB by the LHb-VTA circuit}

Previous studies have found that the LHb receives direct excitatory and inhibitory inputs from the EP (Shabel et al., 2012, 2014; Wallace et al., 2017), and our results showed that c-fos expression in the $\mathrm{LHb}$ exhibited a significant increase at $4 \mathrm{~h}$ after muscimol injection into the EP (Fig. $8 B, D$ ). To further investigate the role of the LHb in SIB, we injected muscimol into the LHb $20 \mathrm{~min}$ before the EP injection. The animals showed a significantly lower wound-occurrence ratio $\left[F_{(2,32)}=15.722, p<0.001\right.$; i.lhb.(sal) + i.ep.(mus-1.0) vs i.lhb.(mus-1.0) + i.ep.(mus-1.0): $p=0.001$; i.lhb.(mus-1.0) + i.ep.(sal) vs i.lhb.(mus-1.0) + i.ep.(mus-1.0): $p=0.049$; Fig. $9 A]$ and severity score $\left[F_{(2,32)}=10.521, p<0.001\right.$; i.lhb.(sal) + i.ep.(mus-1.0) vs i.lhb.(mus-1.0) + i.ep.(mus-1.0): $p=0.005$; i.lhb.(mus-1.0) + i.ep.(sal) vs i.lhb.(mus-1.0) + i.ep. (mus-1.0): $p=0.117$; Fig. $9 B$ ] compared with EP-injected animals with saline injection into the LHb, indicating that the $\mathrm{LHb}$ is indeed an important node downstream of EP in the induction of SIB.

The LHb sends glutamatergic projections to the VTA. This innervates primarily GABAergic neurons, which in turn inhibit the dopaminergic neurons in the VTA (Fig. 9C; Brinschwitz et al., 2010; Russo and Nestler, 2013). Previous studies have shown that dopaminergic modulation plays an important role in synaptic plasticity and reward responses (Zhang et al., 2009; BrombergMartin et al., 2010). In addition, dysregulation of the dopamine system can induce SIB in patients and in animal models (Schroeder et al., 2001). When examining neural activity in the VTA in muscimol-injected rats, we found that the number of c-fospositive cells was significantly increased $4 \mathrm{~h}$ after the injection (drug: $F_{(1,37)}=37.026, p<0.001$; time: $F_{(1,37)}=6.242, p=0.017$; drug $\times$ time interaction: $F_{(1,37)}=11.860, p=0.001$; sal vs mus1.0, 1 h: $p=0.065 ; 4$ h: $p<0.001 ; 1$ vs 4 h, sal: $p=0.541$; mus- 1.0 : $p<0.001$; Fig. $9 D, E$ ). Combined with immunostaining of $\mathrm{TH}$, 
A
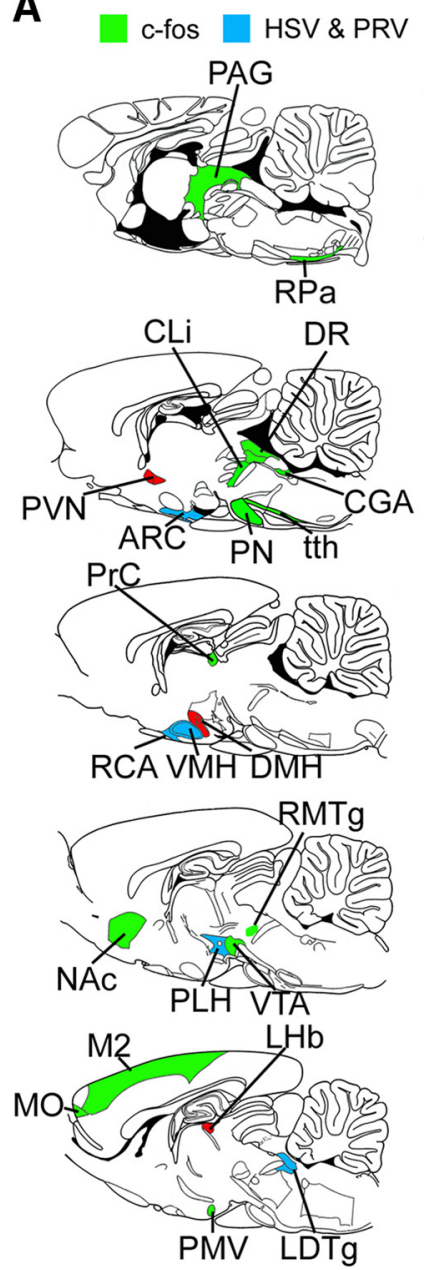

HSV \& PRV \& c-fos
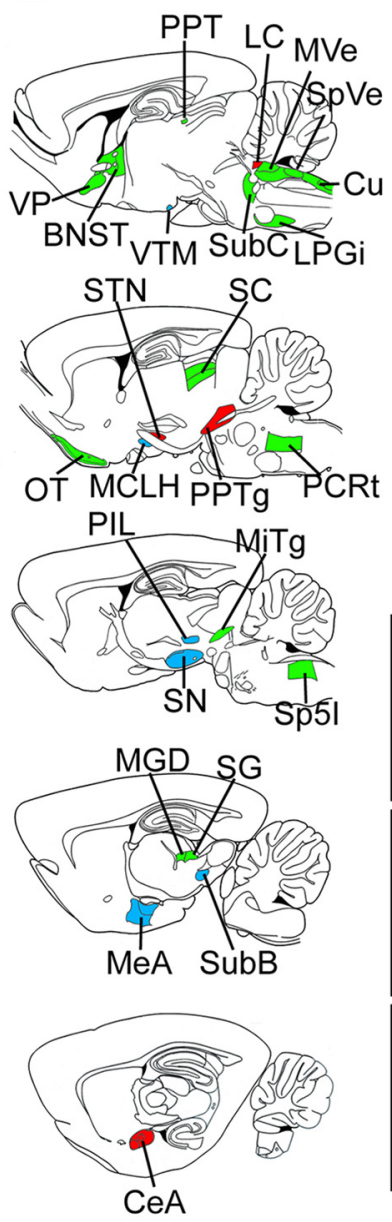

B
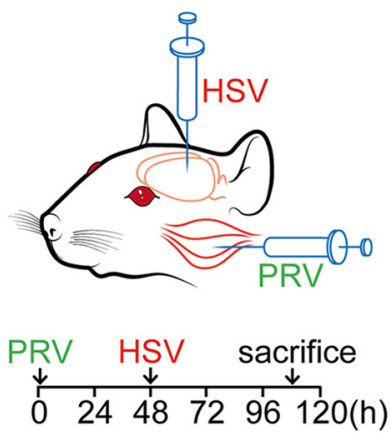

C
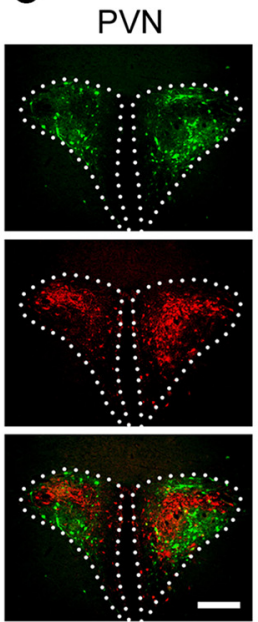

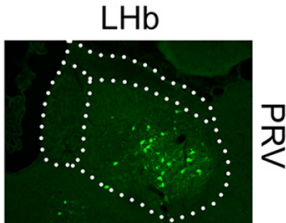

꾼
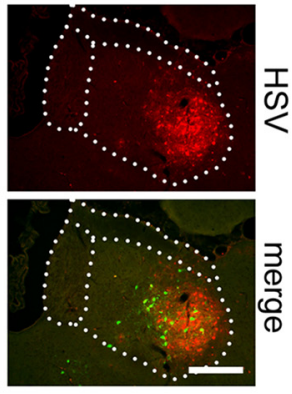

Figure 7. Brain areas showed strong coinfection of PRV and HSV and increased c-fos expression during SIB. A, Activity and connectivity maps of SIB-related brain areas. Green, Brain areas with relatively strong increase in c-fos signals in the mus- $1.0-4 \mathrm{~h}$ group compared with the sal- $4 \mathrm{~h}$ groups; blue, brain areas with relatively strong coinfection of HSV and PRV signals; red, brain areas with relatively strong increases in both c-fos signals and viral coinfection signals. B, Schematic diagram of HSV and PRV injection. C, Representative of viral tracing signals in the PVN and the LHb. Green, PRV signals; red, HSV signals; yellow, merged PRV and HSV signals. ARC, Arcuate hypothalamic nucleus; BNST, bed nucleus of the stria terminalis; CGA, central gray, $\alpha$ part; CLi, caudal linear nucleus of the raphe; $\mathrm{Cu}$, cuneate nucleus; DMH, dorsomedial hypothalamic nucleus; DR, dorsal raphe nucleus; LDTg, laterodorsal tegmental nucleus; LPGi, lateral paragigantocellular nucleus; M2, secondary motor cortex; MCLH, magnocellular nucleus of lateral hypothalamus; MeA, medial amygdala nucleus; MGD, medial geniculate nucleus, dorsal part; MiTg, microcellular tegmental nucleus; MO, medial orbital cortex; MVe, medial vestibular nucleus; NAc, nucleus accumbens; OT, olfactory tubercle; PAG, periaqueductal gray; PCRt, parvocellular reticular nucleus; PIL, posterior intralaminar thalamic nuclei; PLH, peduncular part of lateral hypothalamus; PMV, ventral part of premammillary nucleus; PN, pontine nuclei; PPT, posterior pretectal nucleus; PPTg, pedunculopontine tegmental nucleus; PrC, precommissural nucleus; RCA, retrochiasmatic area; RMTg, rostromedial tegmental nucleus; RPa, raphe pallidus nucleus; $\mathrm{SC}$, superior colliculus; $\mathrm{SG}$, suprageniculate thalamic nucleus; Sp5I, interpolar part of spinal trigeminal nucleus; SpVe, spinal vestibular nucleus; STN, subthalamic nucleus; SubB, subbrachial nucleus; SubC, subcoeruleus nucleus; tth, trigeminothalamic tract; VMH, ventromedial hypothalamic nucleus; VP, ventral pallidum; VTM, ventral tuberomammillary nucleus.

we found that the c-fos expression of both TH-positive dopaminergic neurons and TH-negative neurons, most of which are known to be GABAergic (Yamaguchi et al., 2007; Nair-Roberts et al., 2008; Margolis et al., 2012), increased significantly at $4 \mathrm{~h}$ after muscimol injection (cell type: $F_{(2,75)}=143.986, p<0.001$; time: $F_{(1,75)}=19.849, p<0.001$; cell type $\times$ time interaction: $F_{(2,75)}=$ $5.272, p=0.007$; mus-1.0-1 h vs mus-1.0-4 h: $\mathrm{c}-\mathrm{fos}^{+}-\mathrm{TH}^{+}: p=$ 0.018 ; c-fos ${ }^{+}-\mathrm{TH}^{-}: p<0.001$; total $\mathrm{TH}^{+}: p=0.721$; Fig. $\left.9 F, G\right)$. We also noticed that the ratio between the number of $\mathrm{c}^{-}$fos ${ }^{+}-$ $\mathrm{TH}^{+}$cells and that of c-fos ${ }^{+}-\mathrm{TH}^{-}$cells in the VTA decreased at $4 \mathrm{~h}\left(t_{(25)}=2.099, p=0.023\right.$; Fig. $\left.9 H\right)$.

To evaluate the possible involvement of VTA activity in SIB, we first blocked GABAergic inhibition by injecting $\mathrm{GABA}_{\mathrm{A}}$ receptor antagonist BMI into the VTA 30 min before the EP injection. The treatment significantly reduced the wound-occurrence ratio $\left[F_{(4,55)}=16.053, p<0.001\right.$; i.vta.(sal) + i.ep.(mus-1.0) vs i.vta.(BMI) + i.ep.(mus-1.0): $p<$
0.001; i.vta.(BMI) + i.ep.(sal) vs i.vta.(BMI) + i.ep.(mus1.0): $p=0.005$; Fig. $9 I]$ and severity score $\left[F_{(4,55)}=16.141\right.$, $p<0.001$; i.vta.(sal) + i.ep.(mus-1.0) vs i.vta.(BMI) + i.ep. (mus-1.0): $p<0.001$; i.vta.(BMI) + i.ep.(sal) vs i.vta.(BMI) + i.ep.(mus-1.0): $p=0.011 ;$ Fig. 9J). Intriguingly, muscimol injection into the VTA also caused a significant decrease in the wound-occurrence ratio $\left[F_{(4,55)}=16.053, p<0.001\right.$; i.vta. (sal) + i.ep.(mus-1.0) vs i.vta.(mus-1.0) + i.ep.(mus-1.0): $p<0.001$; i.vta.(mus-1.0) + i.ep.(sal) vs i.vta.(mus-1.0) + i.ep.(mus-1.0): $p=0.069$; Fig. $9 I]$ and severity score $\left[F_{(4,55)}=16.141, p<0.001\right.$; i.vta.(sal) + i.ep.(mus-1.0) vs i.vta.(mus-1.0) + i.ep.(mus-1.0): $p<0.001$; i.vta.(mus-1.0) + i.ep.(sal) vs i.vta.(mus-1.0) + i.ep.(mus-1.0): $p=0.309$; Fig. 9J]. Figures 10 and 11 show the EP, $\mathrm{LHb}$, and VTA injection sites in all the animals used in Figure 9. These results indicate that the LHb-VTA circuit plays a key role in the induction and regulation of SIB. 
A

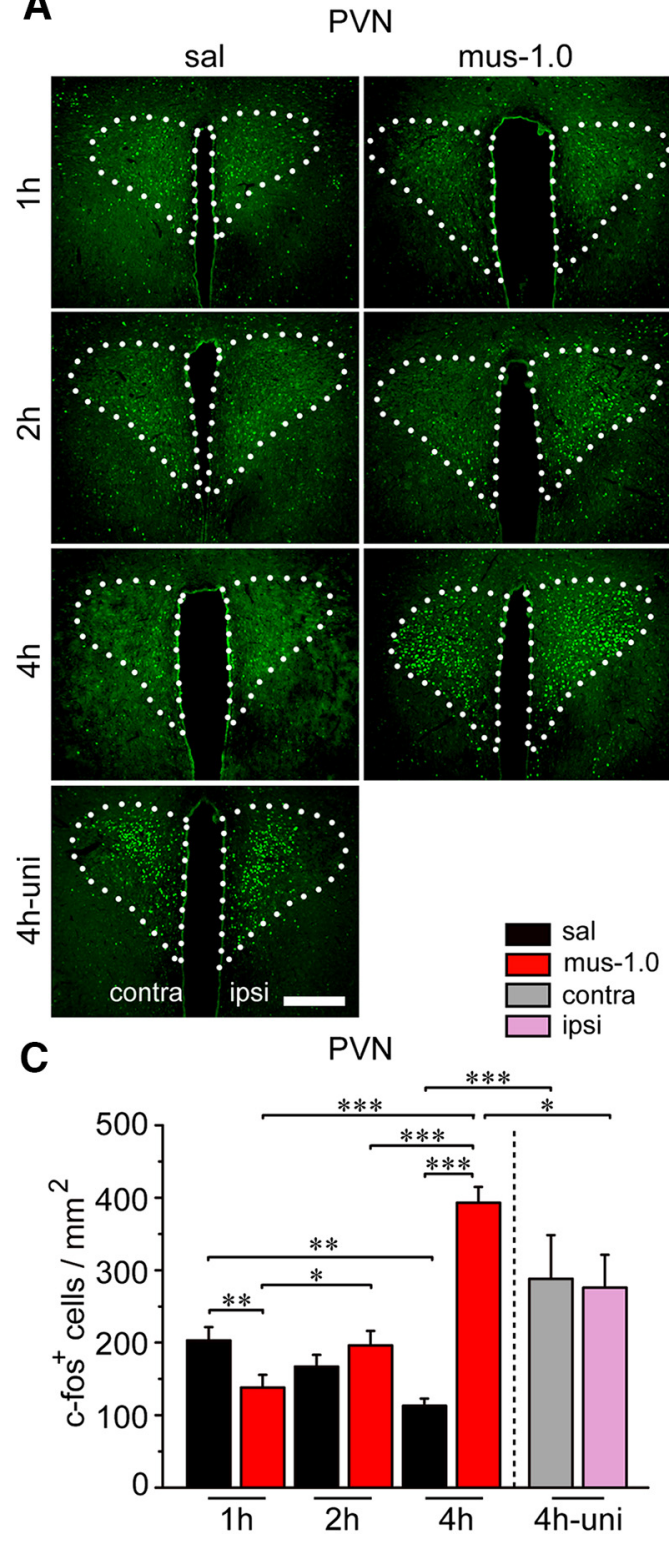

B

LHb
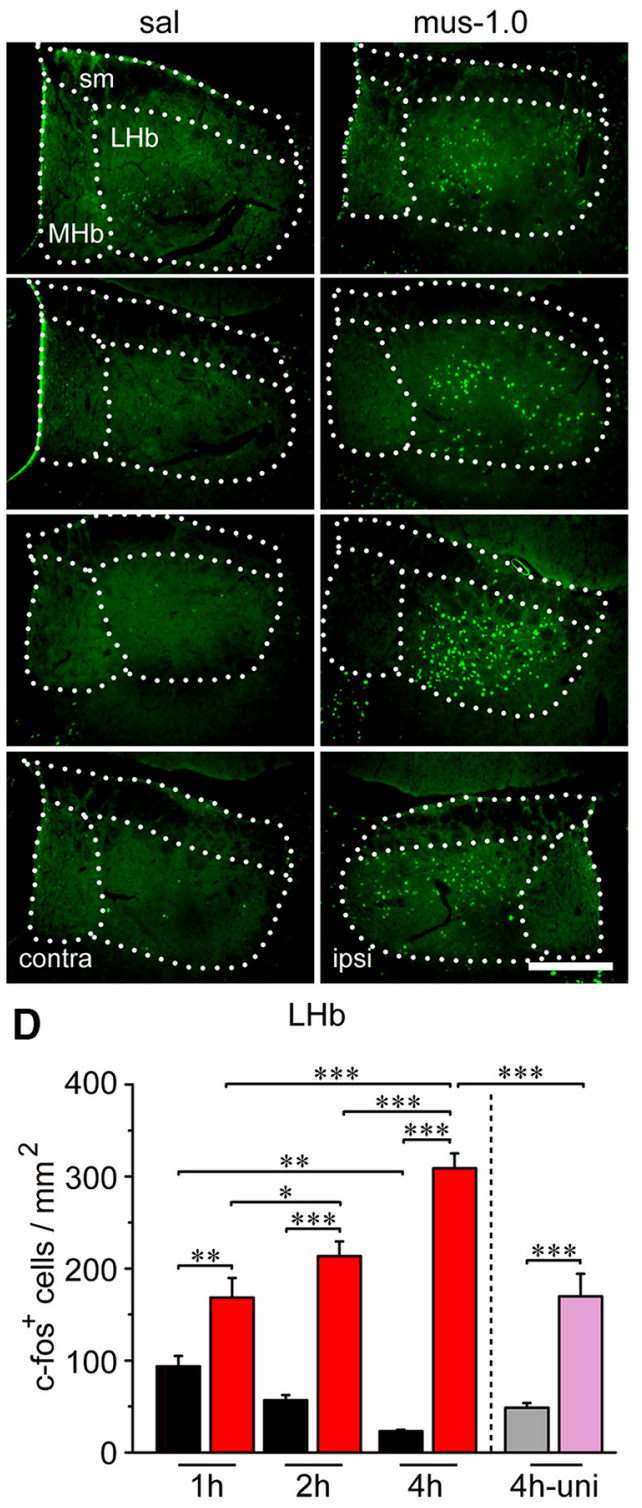

Figure 8. The PVN and the LHb showed increased c-fos expression during SIB. $\boldsymbol{A}, \boldsymbol{B}$, Representative c-fos immunostaining in the PVN $(\boldsymbol{A})$ and in the $L \mathrm{Lb}(\boldsymbol{B})$ at 1,2 , and $4 \mathrm{~h}$ after muscimol injection and $4 \mathrm{~h}$ after unilateral muscimol injection (ipsi, the side ipsilateral to the muscimol injection side; contra, the side contralateral to the muscimol injection site). $C, D$, The density of $c$-fos-positive cells in the PVN $(\boldsymbol{C})$ and the LHb (D). $n=3-5$ animals per group, six slices for each animal. Scale bars, $300 \mu \mathrm{m} .{ }^{*} p<0.05$, ${ }^{* *} p<0.01$, ${ }^{* * *} p<0.001$ for all figures.

\section{Discussion}

Synergistic effects of motor and emotional dysfunctions in SIB We have established a rat model of SIB by disrupting the neural activity in the EP. Such SIB could be modulated by the level of corticosterone and activity in the LHb-VTA circuit. The thalamus and LHb, two major output targets of the EP, play critical roles in motor and emotional functions (Kha et al., 2000; Li et al., 2013). Previous studies have shown that the GPi, the primate homolog of the EP, is associated with movement disorders, such as dystonia and obsessive-compulsive disorder (Zhuang et al., 2004; Nair et al., 2014). Moreover, both the EP in rats and the GPi in primates have roles in aversion encoding (Hong and Hikosaka, 2008; Shabel et al., 2012) and the GPi in humans is involved in emotional disorders, such as major depression disorder, schizophrenia, and suicide (Laplane et al., 1989; Burkhard et al., 2004; Foncke et al., 2006). Therefore, the SIB induced by the disruption of activity in the EP could be due to the synergistic effects of motor and emotional dysfunctions. Indeed, rats with EP impairment showed both stereotypic movements and enhanced corticosterone levels, together with increased neural activity in several stress-related brain areas, such as the PVN, LHb, CeA, and LC. By altering the corticosterone signaling or the activity of the LHbVTA circuit, we found that the wound occurrence could be significantly reduced but never fully blocked, which also indicates the existence of parallel pathways, besides the stress-response system, contributing to SIB. Thus, whereas these emotion-related circuits and signaling systems are potential targets for the intervention of this behavior, it is possible that total rescue of SIB in this model will require coordinated regulation of both the stressresponse systems and the thalamus-cortex-striatum pathway.

\section{Delayed onset of SIB}

Intriguingly, after muscimol injection into the EP, there was always a 40-60 min delay before the onset of stereotypic head 


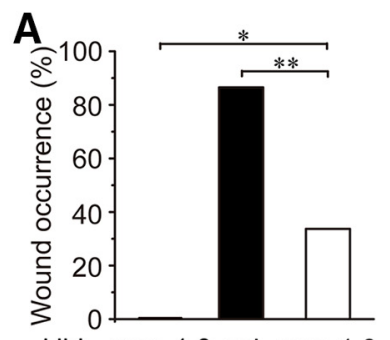

i.lhb. mus-1.0 sal mus-1.0

i.ep. sal $\overleftrightarrow{\text { mus-1.0 }}$

\section{D}

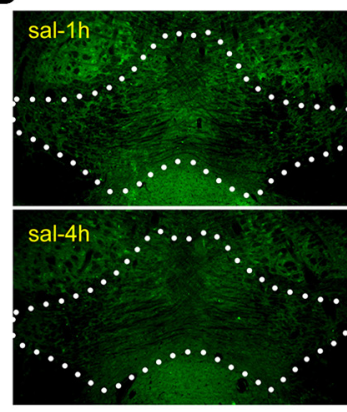

B

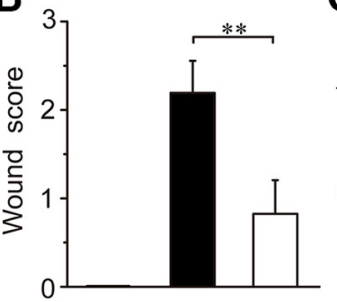

i.lhb. mus-1.0 sal mus-1.0 i.ep. sal $\overleftrightarrow{\text { mus-1.0 }}$
C

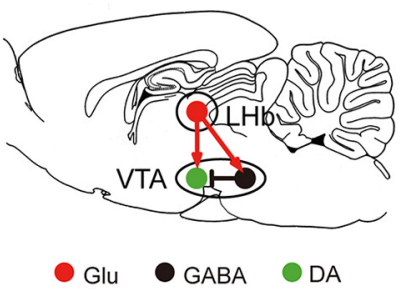

E

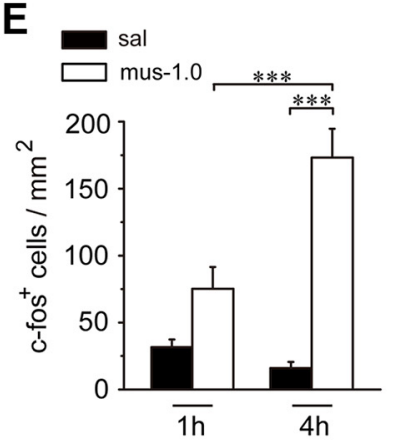

G

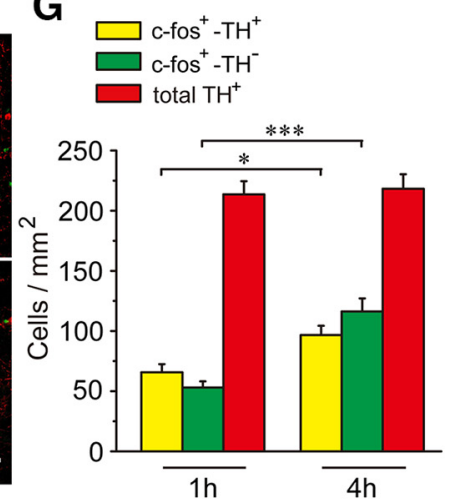

$\mathbf{J}$

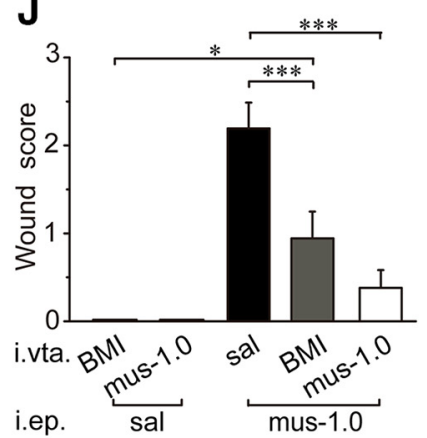

Figure 9. Modulation of SIB by regulating the activity of the LHb-VTA circuit. $A, B$, The wound-occurrence ratio $(A)$ and the severity $(\boldsymbol{B})$ were significantly decreased by injection of muscimol into the $L \mathrm{Hb}[$ i.l.hb.(mus-1.0) + i.ep.(sal), $n=9$; i.lhb.(sal) + i.ep.(mus-1.0), $n=14$; i.lhb.(mus-1.0) + i.ep.(mus-1.0), $n=12$ ]. i.lhb., LHb injection. C, Schematic diagram of the direct projections from the LHb to the VTA. Red circle, Glutamatergic neurons; black circle, GABAergic neurons; green circle, dopaminergic neurons. $\boldsymbol{D}$, Representative images of c-fos immunostaining in the VTA. Scale bar, $300 \mu \mathrm{m}$. Green, c-fos signals. sal- $1 \mathrm{~h}, 1 \mathrm{~h}$ after bilateral injection with saline; sal- $4 \mathrm{~h}, 4 \mathrm{~h}$ after bilateral injection with saline; mus-1.0-1 $\mathrm{h}, 1 \mathrm{~h}$ after bilateral injection with $1.0 \mu \mathrm{g}$ of muscimol per site; mus-1.0-4 h, $4 \mathrm{~h}$ after bilateral injection with $1.0 \mu \mathrm{g}$ of muscimol per site. $\boldsymbol{E}$, The density of c-fos-positive cells in the VTA ( $n=3-4$ animals per group, 3 slices for each animal). c-fos expression in VTA $4 \mathrm{~h}$ after the muscimol injection was significantly higher than that $1 \mathrm{~h}$ after the muscimol injection or $4 \mathrm{~h}$ after the saline injection. $\boldsymbol{F}$, Representative c-fos and TH immunostaining in the VTA. Green, c-fos signals; red, TH signals; yellow, merged c-fos and TH signals. Scale bar, $100 \mu \mathrm{m}$. G, Both c-fos-positive, TH-positive $\left(\mathrm{c}_{-} \mathrm{fos}^{+}{ }^{-\mathrm{TH}^{+}}\right)$and c-fos-positive, TH-negative $\left(\mathrm{c}-\mathrm{fos}^{+}\right.$TH $^{-}$) VTA neurons increased significantly at $4 \mathrm{~h}$ after muscimol injection ( $n=4-5$ animals per group, 3 slices for each animal). $\boldsymbol{H}$, The ratio between the number of $\mathrm{c}-\mathrm{fos}^{+}-\mathrm{TH}^{+}$cells and $\mathrm{c}-\mathrm{fos}^{+}-\mathrm{TH}^{-}$cells in the mus-1.0-4 $\mathrm{h}$ group was significantly lower than that in the mus-1.0-1 h group. $I, J$, Blockade or excitation of GABAergic transmission in the VTA by GABA receptor antagonist BMI or muscimol (mus) significantly decreased the wound-occurrence ratio $(I)$ and the wound severity $[J ;$ i.vta. movements (Fig. $1 F$ ), a phenomenon also observed in rats with SIB induced by muscimol injection into the SN (Baumeister and Frye, 1984). Because off-target injections near the EP generally failed to induce SIB (Fig. 3), it is unlikely that the delay is due to diffusion of muscimol to these surrounding areas. Instead, we suspect that the slow development of abnormal behavior reflects changes in dynamic interactions among complex circuitry and hormonal systems. This complexity may also underlie the observed individual variability among animals in their susceptibility to similar SIB-inducing treatments, e.g., bilateral injection of $0.2 \mu \mathrm{g}$ of muscimol into the EP (Fig. 4B). The many brain areas identified by our SIB activity and connectivity maps provide potential candidates for future investigation of this complexity.

\section{Activity in the LHb-VTA pathway during SIB}

Previous studies reported that the EP sends GABAergic projections to the thalamus, whereas glutamate/GABA coreleasing neurons and a rare population of purely glutamatergic neurons in the EP send projections to the $\mathrm{LHb}$ (Wallace et al., 2017). LHb-projecting EP neurons showed encoding properties similar to those of LHb neurons in expecting "antireward" signals (Hong and Hikosaka, 2008; Shabel et al., 2012; StephensonJones et al., 2016), and the balance of glutamate/GABA corelease was changed in depression and cocaine-withdrawal rodents (Shabel et al., 2014; Meye et al., 2016). It is thus possible that muscimol injection into the EP would silence its neural activity and therefore remove the inhibition to the $\mathrm{LHb}$ from the glutamate/ GABA coreleasing neurons in the EP, leaving $\mathrm{LHb}$ neurons more readily activated by other inputs. This scenario is consistent with the increase of c-fos expression in the $\mathrm{LHb}$ following the muscimol injection into the EP (Fig. $8 B, D$ ).

Driven by the excitatory inputs from the LHb (Russo and Nestler, 2013), neural activity in the VTA also increased in rats showing SIB, both in TH-positive dopaminergic neurons and in TH-negative neurons, which are most likely GABAergic

\footnotetext{
$($ BMI) + i.ep.(sal), $n=9$; i.vta.(mus-1.0) + i.ep.(sal), $n=$ 11; i.vta. (sal) + i.ep.(mus-1.0), $n=15$; i.vta.(BMI) + i.ep.(mus-1.0), $n=14$; i.vta.(mus-1.0) + i.ep.(mus-1.0), $n=11$.i.vta., VTA injection]. ${ }^{*} p<0.05,{ }^{* *} p<0.01,{ }^{* * *} p<$ 0.001 for all figures.
} 
A

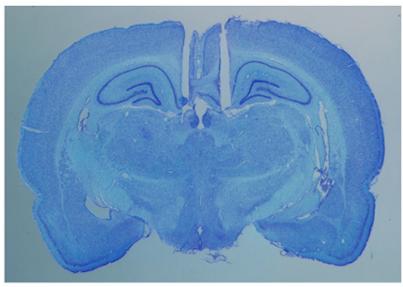

B

\section{$\circ$ no wound \\ $\circ$ wounds}

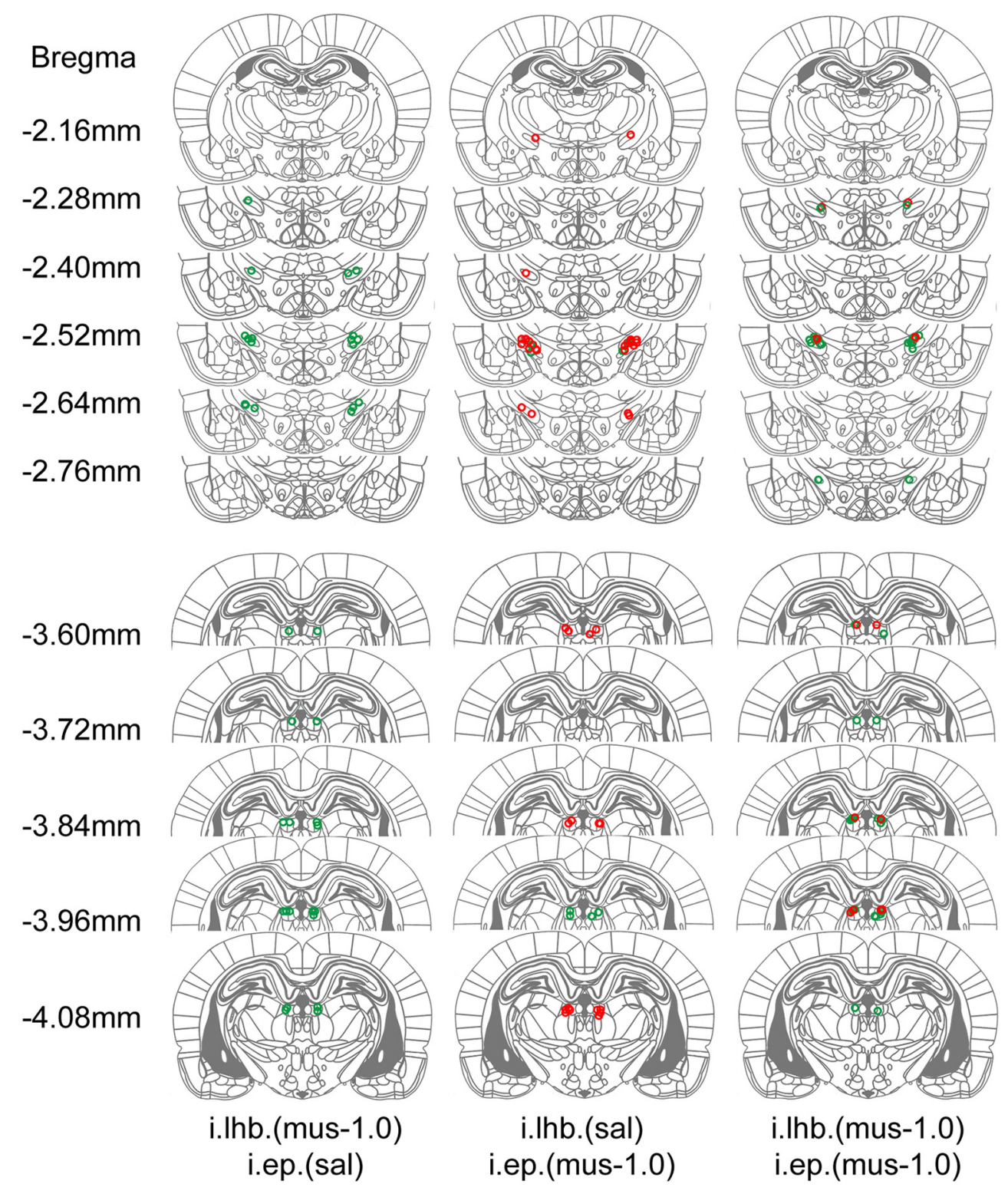

Figure 10. Locations of EP and LHb injection sites in the animals used in Figure $9 A, B$. $A$, An example brain slice with Nissl staining showing cannulae locations in the $L H b$. $B$, Summary of verified injection sites shown on a brain atlas (red, rats with wounds; green, rats with no wounds).

neurons (Fig. 9G,H). Interestingly, the GABAergic neurons in the VTA are also known to receive direct inputs from the PVN (Beier et al., 2015). Thus, the increased activity in the PVN could also contribute to the enhanced activation of GABAergic neurons in the VTA during SIB (Figs. $8 A, C, 9 G, H$ ). Notably, both activity inhibition by muscimol and disinhibition by BMI injected into the VTA partially rescued SIB (Fig. 9I,J), suggesting that an "optimal" activation level of VTA dopaminergic neurons might be required to positively modulate the behavior. This is reminiscent of the inverted-U-shaped curve of dopaminergic modulation of other functional circuits, such as the prefrontal cortex during working memory tasks (Vijayraghavan et al., 2007). In any case, our results suggest that the LHb-VTA pathway may be a potential therapeutic target for treating SIB and related behavioral abnormalities. For example, it may be a promising target in patients or animal models with autism or Lesch-Nyhan disease, who have 
A

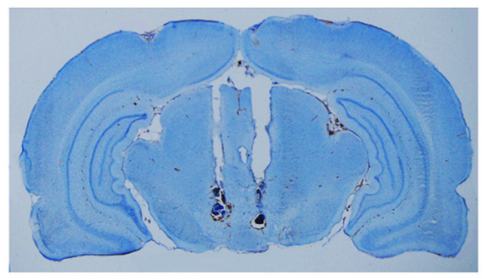

o no wound o wounds

B

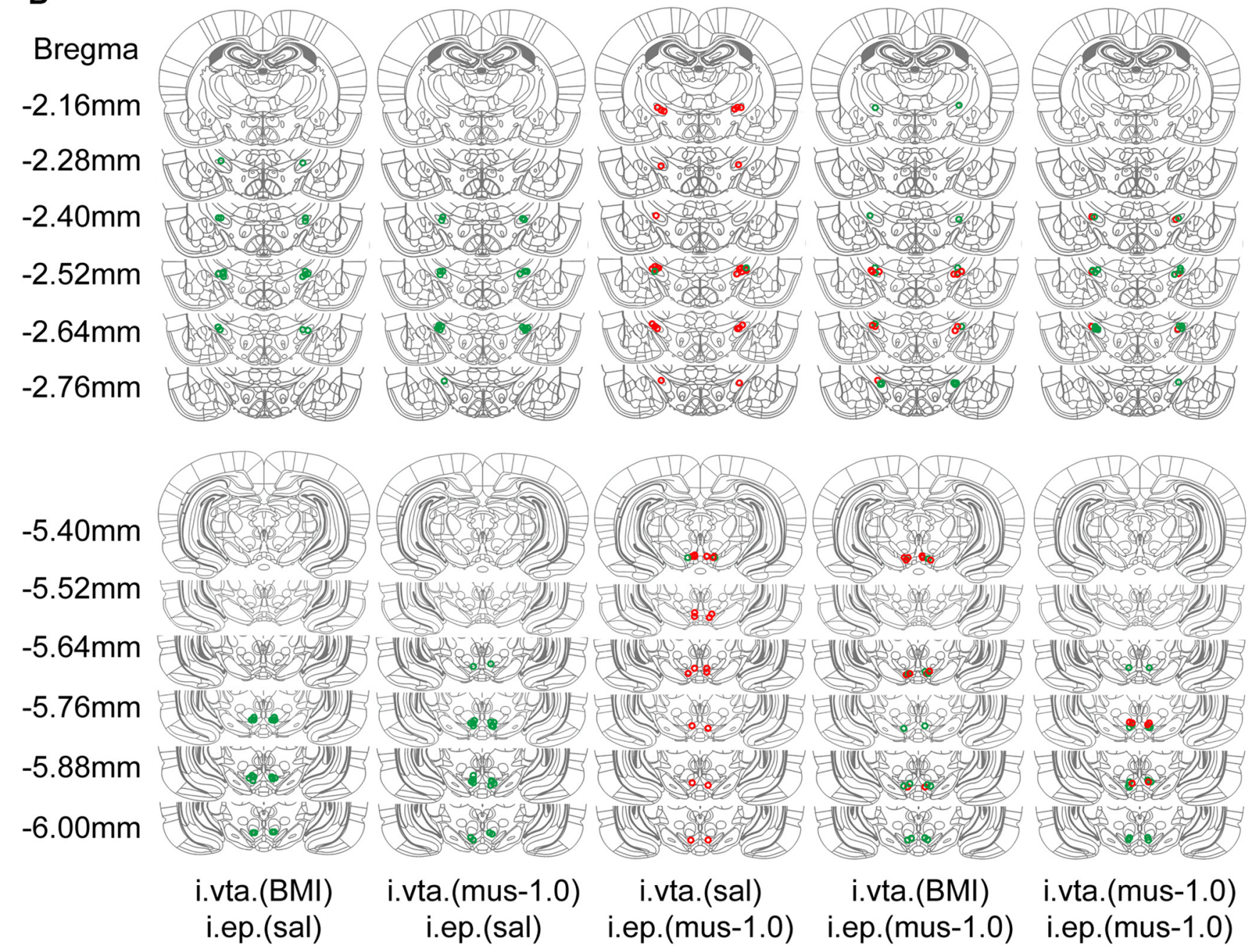

Figure 11. Locations of EP and VTA injection sites in the animals used in Figure $9 /, J$. $A$, An example brain slice with Niss/ staining showing cannulae locations in the VTA. $\boldsymbol{B}$, Summary of verified injection sites shown on a brain atlas (red, rats with wounds; green, rats with no wounds).

been found to have decreased concentrations of dopamine and metabolites in various brain areas, including the frontal cortex, basal ganglia, and ventral tegmental complex (Jinnah et al., 1994; Ernst et al., 1996, 1997; Liu et al., 2014).

In conclusion, we have established a circuit-specific rat model of SIB that is acutely induced by disruption of EP activity. With this model, we demonstrate that SIB can be modulated by stress and the glucocorticoid system. Furthermore, we have identified brain areas by screening the relevant activity and connectivity maps in our SIB model, especially the LHb-VTA circuit, in the induction and modulation of SIB. Further studies on more brain areas, perhaps with other methods of induction, should reveal new insights into the complex circuit mechanisms underlying
SIB, and could lead to new therapeutic approaches for the treatment of related disorders.

\section{References}

Anderson LT, Ernst M (1994) Self-injury in Lesch-Nyhan disease. J Autism Dev Disord 24:67-81. CrossRef Medline

Baumeister AA, Frye GD (1984) Self-injurious behavior in rats produced by intranigral microinjection of GABA agonists. Pharmacol Biochem Behav 21:89-95. CrossRef Medline

Beckett C, Bredenkamp D, Castle J, Groothues C, O’Connor TG, Rutter M, Rutter M (2002) Behavior patterns associated with institutional deprivation: a study of children adopted from Romania. J Dev Behav Pediatr 23:297-303. CrossRef Medline

Beier KT, Steinberg EE, DeLoach KE, Xie S, Miyamichi K, Schwarz L, Gao XJJ, 
Kremer EJ, Malenka RC, Luo L (2015) Circuit architecture of VTA dopamine neurons revealed by systematic input-output mapping. Cell 162: 622-634. CrossRef Medline

Bentley KH, Cassiello-Robbins CF, Vittorio L, Sauer-Zavala S, Barlow DH (2015) The association between nonsuicidal self-injury and the emotional disorders: a meta-analytic review. Clin Psychol Rev 37:72-88. CrossRef Medline

Bodfish JW (2007) Stereotypy, self-injury, and related abnormal repetitive behaviors. In: Handbook of intellectual and developmental disabilities (Jacobson JW, Mulick JA, Rojahn J, eds), pp 481-505. New York, NY: Springer US.

Briere J, Gil E (1998) Self-mutilation in clinical and general population samples: prevalence, correlates, and functions. Am J Orthopsychiatry 68:609620. CrossRef Medline

Brinschwitz K, Dittgen A, Madai VI, Lommel R, Geisler S, Veh RW (2010) Glutamatergic axons from the lateral habenula mainly terminate on GABAergic neurons of the ventral midbrain. Neuroscience 168:463-476. CrossRef Medline

Bromberg-Martin ES, Matsumoto M, Hikosaka O (2010) Dopamine in motivational control: rewarding, aversive, and alerting. Neuron 68:815-834. CrossRef Medline

Burkhard PR, Vingerhoets FJ, Berney A, Bogousslavsky J, Villemure JG, Ghika J (2004) Suicide after successful deep brain stimulation for movement disorders. Neurology 63:2170-2172. CrossRef Medline

Chan J, Ni Y, Zhang P, Zhang J, Chen Y (2017) D1-like dopamine receptor dysfunction in the lateral habenula nucleus increased anxiety-like behavior in rat. Neuroscience 340:542-550. CrossRef Medline

Curin JM, Terzić J, Petković ZB, Zekan L, Terzić IM, Susnjara IM (2003) Lower cortisol and higher ACTH levels in individuals with autism. J Autism Dev Disord 33:443-448. CrossRef Medline

Davenport MD, Lutz CK, Tiefenbacher S, Novak MA, Meyer JS (2008) A rhesus monkey model of self-injury: effects of relocation stress on behavior and neuroendocrine function. Biol Psychiatry 63:990-996. CrossRef Medline

Devine DP (2012) Animal models of self-injurious behaviour: an overview. In: Psychiatric disorders: methods and protocols (Kobeissy FH, ed), pp 65-84. New York, NY: Humana.

Devine DP (2014) Self-injurious behaviour in autistic children: a neurodevelopmental theory of social and environmental isolation. Psychopharmacology (Berl) 231:979-997. CrossRef Medline

Dum RP, Strick PL (2013) Transneuronal tracing with neurotropic viruses reveals network macroarchitecture. Curr Opin Neurobiol 23:245-249. CrossRef Medline

Ernst M, Zametkin AJ, Matochik JA, Pascualvaca D, Jons PH, Hardy K, Hankerson JG, Doudet DJ, Cohen RM (1996) Presynaptic dopaminergic deficits in Lesch-Nyhan disease. N Engl J Med 334:1568-1572. CrossRef Medline

Ernst M, Zametkin AJ, Matochik JA, Pascualvaca D, Cohen RM (1997) Low medial prefrontal dopaminergic activity in autistic children. Lancet 350: 638. CrossRef Medline

Favaro A, Ferrara S, Santonastaso P (2007) Self-injurious behavior in a community sample of young women: relationship with childhood abuse and other types of self-damaging behaviors. J Clin Psychiatry 68:122-131. CrossRef Medline

Fay RA, Norgren R (1997) Identification of rat brainstem multisynaptic connections to the oral motor nuclei using pseudorabies virus. III. Lingual muscle motor systems. Brain Res Rev 25:291-311. CrossRef Medline

Foncke EM, Schuurman PR, Speelman JD (2006) Suicide after deep brain stimulation of the internal globus pallidus for dystonia. Neurology 66: 142-143. CrossRef Medline

Herman JP, Cullinan WE (1997) Neurocircuitry of stress: central control of the hypothalamo-pituitary-adrenocortical axis. Trends Neurosci 20:7884. CrossRef Medline

Hikosaka O (2010) The habenula: from stress evasion to value-based decision-making. Nat Rev Neurosci 11:503-513. CrossRef Medline

Holly EN, Miczek KA (2016) Ventral tegmental area dopamine revisited: effects of acute and repeated stress. Psychopharmacology (Berl) 233:163186. CrossRef Medline

Hong S, Hikosaka O (2008) The globus pallidus sends reward-related signals to the lateral habenula. Neuron 60:720-729. CrossRef Medline

Jinnah HA, Wojcik BE, Hunt M, Narang N, Lee KY, Goldstein M, Wamsley JK, Langlais PJ, Friedmann T (1994) Dopamine deficiency in a genetic mouse model of Lesch-Nyhan disease. J Neurosci 14:1164-1175. CrossRef Medline

Kha HT, Finkelstein DI, Pow DV, Lawrence AJ, Horne MK (2000) Study of projections from the entopeduncular nucleus to the thalamus of the rat. J Comp Neurol 426:366-377. CrossRef Medline

Kirby ED, Muroy SE, Sun WG, Covarrubias D, Leong MJ, Barchas LA, Kaufer D (2013) Acute stress enhances adult rat hippocampal neurogenesis and activation of newborn neurons via secreted astrocytic FGF2. Elife 2:e00362. CrossRef Medline

Klonsky ED (2007) The functions of deliberate self-injury: a review of the evidence. Clin Psychol Rev 27:226-239. CrossRef Medline

Krukoff TL (1999) C-fos expression as a marker of functional activity in the brain. In: Cell neurobiology techniques (Boulton AA, Baker GB, Bateson AN, eds), pp 213-230. New York, NY: Humana.

Laplane D, Levasseur M, Pillon B, Dubois B, Baulac M, Mazoyer B, Tran Dinh S, Sette G, Danze F, Baron JC (1989) Obsessive-compulsive and other behavioural changes with bilateral basal ganglia lesions. A neuropsychological, magnetic resonance imaging and positron tomography study. Brain 112:699-725. CrossRef Medline

Li K, Zhou T, Liao L, Yang Z, Wong C, Henn F, Malinow R, Yates JR 3rd, Hu $\mathrm{H}$ (2013) $\beta$ CaMKII in lateral habenula mediates core symptoms of depression. Science 341:1016-1020. CrossRef Medline

Liu A, Li A, Zhang H, Zhang Z (2014) Decreased dopamine concentrations in the frontal cortex after ablative surgeries in patients exhibiting selfinjurious behavior: a microdialysis study. J Neuropsychiatry Clin Neurosci 26:382-385. CrossRef Medline

Margolis EB, Toy B, Himmels P, Morales M, Fields HL (2012) Identification of rat ventral tegmental area GABAergic neurons. PLoS One 7:e42365. CrossRef Medline

Meye FJ, Soiza-Reilly M, Smit T, Diana MA, Schwarz MK, Mameli M (2016) Shifted pallidal co-release of GABA and glutamate in habenula drives cocaine withdrawal and relapse. Nat Neurosci 19:1019-1024. CrossRef Medline

Muehlmann AM, Lewis MH (2012) Abnormal repetitive behaviours: shared phenomenology and pathophysiology. J Intellect Disabil Res 56: 427-440. CrossRef Medline

Muehlmann AM, Kies SD, Turner CA, Wolfman S, Lewis MH, Devine DP (2012) Self-injurious behaviour: limbic dysregulation and stress effects in an animal model. J Intellect Disabil Res 56:490-500. CrossRef Medline

Nair G, Evans A, Bear RE, Velakoulis D, Bittar RG (2014) The anteromedial GPi as a new target for deep brain stimulation in obsessive compulsive disorder. J Clin Neurosci 21:815-821. CrossRef Medline

Nair-Roberts RG, Chatelain-Badie SD, Benson E, White-Cooper H, Bolam JP, Ungless MA (2008) Stereological estimates of dopaminergic, GABAergic and glutamatergic neurons in the ventral tegmental area, substantia nigra and retrorubral field in the rat. Neuroscience 152:10241031. CrossRef Medline

Okun MS, Green J, Saben R, Gross R, Foote KD, Vitek JL (2003) Mood changes with deep brain stimulation of STN and GPi: results of a pilot study. J Neurol Neurosurg Psychiatry 74:1584-1586. CrossRef Medline

Park H, Rhee J, Park K, Han JS, Malinow R, Chung C (2017) Exposure to stressors facilitates long-term synaptic potentiation in the lateral habenula. J Neurosci 37:6021-6030. CrossRef Medline

Peça J, Feliciano C, Ting JT, Wang W, Wells MF, Venkatraman TN, Lascola CD, Fu Z, Feng G (2011) Shank3 mutant mice display autistic-like behaviours and striatal dysfunction. Nature 472:437-442. CrossRef Medline

Proulx CD, Hikosaka O, Malinow R (2014) Reward processing by the lateral habenula in normal and depressive behaviors. Nat Neurosci 17:11461152. CrossRef Medline

Russo SJ, Nestler EJ (2013) The brain reward circuitry in mood disorders. Nat Rev Neurosci 14:609-625. CrossRef Medline

Sandman CA, Barron JL, Chicz-DeMet A, DeMet EM (1991) Brief report: plasma $\beta$-endorphin and cortisol levels in autistic patients. J Autism Dev Disord 21:83-87. CrossRef Medline

Schroeder SR, Oster-Granite ML, Berkson G, Bodfish JW, Breese GR, Cataldo MF, Cook EH, Crnic LS, DeLeon I, Fisher W, Harris JC, Horner RH, Iwata B, Jinnah HA, King BH, Lauder JM, Lewis MH, Newell K, Nyhan WL, Rojahn J et al. (2001) Self-injurious behavior: gene-brain-behavior relationships. Ment Retard Dev Disabil Res Rev 7:3-12. CrossRef Medline

Shabel SJ, Proulx CD, Trias A, Murphy RT, Malinow R (2012) Input to the 
lateral habenula from the basal ganglia is excitatory, aversive, and suppressed by serotonin. Neuron 74:475-481. CrossRef Medline

Shabel SJ, Proulx CD, Piriz J, Malinow R (2014) Mood regulation. GABA/ glutamate co-release controls habenula output and is modified by antidepressant treatment. Science 345:1494-1498. CrossRef Medline

Stanek E 4th, Cheng S, Takatoh J, Han BX, Wang F (2014) Monosynaptic premotor circuit tracing reveals neural substrates for oro-motor coordination. Elife 3:e02511. CrossRef Medline

Stephenson-Jones M, Yu K, Ahrens S, Tucciarone JM, van Huijstee AN, Mejia LA, Penzo MA, Tai LH, Wilbrecht L, Li B (2016) A basal ganglia circuit for evaluating action outcomes. Nature 539:289-293. CrossRef Medline

Symons FJ, Sutton KA, Walker C, Bodfish JW (2003) Altered diurnal pattern of salivary substance $P$ in adults with developmental disabilities and chronic self-injury. Am J Ment Retard 108:13-18. CrossRef Medline

Tang H, Wu GS, Xie J, He X, Deng K, Wang H, Xu F, Luo HR (2016) Brain-wide map of projections from mice ventral subiculum. Neurosci Lett 629:171-179. CrossRef Medline

Turner C, Panksepp J, Bekkedal M, Borkowski C, Burgdorf J (1999) Paradoxical effects of serotonin and opioids in pemoline-induced selfinjurious behavior. Pharmacol Biochem Behav 63:361-366. CrossRef Medline

Vijayraghavan S, Wang M, Birnbaum SG, Williams GV, Arnsten AF (2007) Inverted- $\mathrm{U}$ dopamine $\mathrm{D} 1$ receptor actions on prefrontal neurons engaged in working memory. Nat Neurosci 10:376-384. CrossRef Medline
Wallace ML, Saunders A, Huang KW, Philson AC, Goldman M, Macosko EZ, McCarroll SA, Sabatini BL (2017) Genetically distinct parallel pathways in the entopeduncular nucleus for limbic and sensorimotor output of the basal ganglia. Neuron 94:138-152.e5. CrossRef Medline

Wei P, Liu N, Zhang Z, Liu X, Tang Y, He X, Wu B, Zhou Z, Liu Y, Li J, Zhang Y, Zhou X, Xu L, Chen L, Bi G, Hu X, Xu F, Wang L (2015) Processing of visually evoked innate fear by a non-canonical thalamic pathway. Nat Commun 6:6756. CrossRef Medline

Xu L, Holscher C, Anwyl R, Rowan MJ (1998) Glucocorticoid receptor and protein/RNA synthesis-dependent mechanisms underlie the control of synaptic plasticity by stress. Proc Natl Acad Sci U S A 95:3204-3208. CrossRef Medline

Yamaguchi T, Sheen W, Morales M (2007) Glutamatergic neurons are present in the rat ventral tegmental area. Eur J Neurosci 25:106-118. CrossRef Medline

Zemanick MC, Strick PL, Dix RD (1991) Direction of transneuronal transport of herpes-simplex virus-1 in the primate motor system is straindependent. Proc Natl Acad Sci U S A 88:8048-8051. CrossRef Medline

Zhang JC, Lau PM, Bi GQ (2009) Gain in sensitivity and loss in temporal contrast of STDP by dopaminergic modulation at hippocampal synapses. Proc Natl Acad Sci U S A 106:13028-13033. CrossRef Medline

Zhuang P, Li Y, Hallett M (2004) Neuronal activity in the basal ganglia and thalamus in patients with dystonia. Clin Neurophysiol 115:2542-2557. CrossRef Medline 